\title{
FINITE SUBLATTICES OF A FREE LATTICE
}

\author{
BY \\ J. B. NATION ${ }^{1}$
}

\begin{abstract}
Every finite semidistributive lattice satisfying Whitman's condition is isomorphic to a sublattice of a free lattice.
\end{abstract}

Introduction. The aim of this paper is to show that a finite semidistributive lattice satisfying Whitman's condition can be embedded in a free lattice. This confirms a conjecture of Bjarni Jónsson, and indeed our proof will follow the line of approach originally suggested by him in unpublished notes around 1960. This approach was later described in Jónsson and Nation [15], to which the reader is referred for a more complete discussion of the background material and related work than will be given here.

Let us recall some relevant definitions and results. A finite sublattice of a free lattice satisfies Whitman's condition [23]

(W) $a b \leqslant c+d$ iff $a \leqslant c+d$ or $b \leqslant c+d$ or $a b \leqslant c$ or $a b \leqslant d$ and the semidistributive laws introduced by Jónsson [12]

$\left(\mathrm{SD}_{\vee}\right) u=a+b=a+c$ implies $u=a+b c$,

$\left(\mathrm{SD}_{\wedge}\right) u=a b=a c$ implies $u=a(b+c)$.

As in [15], we shall refer to a finite lattice satisfying these three conditions as an $S$-lattice.

We will often use the following (equivalent) form of the semidistributive laws [14].

$$
\begin{aligned}
& \left(\mathrm{SD}_{\vee}\right) u=\sum a_{i}=\sum b_{j} \text { implies } u=\sum_{i} \sum_{j} a_{i} b_{j} \\
& \left(\mathrm{SD}_{\wedge}\right) u=\Pi a_{i}=\Pi b_{j} \text { implies } u=\Pi_{i} \Pi_{j}\left(a_{i}+b_{j}\right)
\end{aligned}
$$

Let $J(L)$ denote the set of nonzero join-irreducible elements in a finite lattice $L$. Every element $p \in J(L)$ has a unique lower cover, which we will denote by $p_{*}$. If $p_{*} \in J(L)$, let $p_{* *}=\left(p_{*}\right)_{*}$. Dually, $M(L)$ denotes the set of nonunit meet-irreducible elements of $L$, and for $y \in M(L), y^{*}>y$. In a finite semidistributive lattice there is a bijection between $J(L)$ and $M(L)$,

$$
p \leftrightarrow \kappa(p) \equiv \sum\left\{x \in L: x \geqslant p_{*} \text { and } x \neq p\right\} .
$$

(In fact, A. Day has shown that this characterizes finite semidistributive lattices [4].) Now $p x=p_{*}$ iff $x \geqslant p_{*}$ and $x \neq p$, and, by $\left(\operatorname{SD}_{\wedge}\right), p \kappa(p)=p_{*}$; th.us $\kappa(p)$ is the largest element in $L$ with this property. Repeatedly we will use the following observations.

Received by the editors November 19, 1980.

1980 Mathematics Subject Classification. Primary 06B25; Secondary 08B20.

'This work was supported in part by the National Science Foundation Grant No. MCS79-01735. 
LEMMA 1.1. If $L$ is a finite semidistributive lattice and $p \in J(L)$, then

(i) $p+\kappa(p)=\kappa(p)^{*}$,

(ii) $x \leqslant \kappa(p)$ iff $p_{*}+x \neq p$.

For a finite semidistributive lattice $L$, we define binary relations $A$ and $B$ on $J(L)$ as follows.

$$
\begin{gathered}
p A q \text { if } q<p \leqslant \kappa(q)^{*}, \\
p B q \text { if } p \neq q, q_{*} \leqslant \kappa(p), q \leqslant \kappa(p) .
\end{gathered}
$$

For technical purposes these relations are further subdivided.

$$
\begin{aligned}
& p A_{1} q \text { if } p A q \text { and } p \kappa(q)>q_{*}, \\
& p A_{2} q \text { if } p A q \text { and } p \kappa(q)=q_{*}, \\
& p B_{1} q \text { if } p B q \text { and } q_{*} \$ p, \\
& p B_{2} q \text { if } p B q \text { and } q_{*}<p_{*} .
\end{aligned}
$$

Note that by Lemma 1.1(ii) we have $p B q$ iff $p \neq q, p \leqslant p_{*}+q_{*}, p \leqslant p_{*}+q$. It follows that if $p B q$, then $p_{*} \$ q$ (whence $p \$ q$ ) and $q \$ p$. Moreover, if $p B q$, then $p \leqslant \kappa(q)$. For otherwise we would have $p+q=p_{*}+q$ and $p+q=p+q_{*}$, whence by $\left(\mathrm{SD}_{\vee}\right) p+q=p_{*}+q_{*}+p q=p_{*}+q_{*}$ (as $p$ and $q$ must be incomparable), while since $q_{*} \leqslant \kappa(p)$ we have $p_{*}+q_{*} \neq p$, a contradiction. Thus the drawings of Figure 1 accurately represent these relations insofar as the joins and meets of the labeled elements are concerned.

Finally, let $C=A \cup B$, i.e., $p C q$ if $p A q$ or $p B q$.

By a cycle in a finite semidistributive lattice, we mean a sequence $\left\langle p_{0}, p_{1}, \ldots, p_{n}\right\rangle$ with $n \geqslant 1$ of join-irreducible elements such that $p_{i} C p_{i+1}$ for $0 \leqslant i<n$, and $p_{n} C p_{0}$. A minimal cycle means one of minimal length in $L$. In particular, a minimal cycle has the property that $p_{i} C p_{j}$ only if $j=i+1$.

Our approach is based on the following result, which combines Theorems 2.1, 6.4 , and 9.3 of [15].

THEOREM 1.2. A finite lattice is embeddable in a free lattice iff it is an $S$-lattice containing no cycle.

What we will show is that no $S$-lattice contains a cycle, so that every $S$-lattice is isomorphic to a sublattice of a free lattice.

For the sake of completeness, let us sketch the proof of the relevant direction of Theorem 1.2, which shows that an $S$-lattice not containing a cycle is in fact projective. These arguments were all contained in Jonsson's original notes. The details may be found in [15].

For $U, V \subseteq L$, we write $V \ll U$ if for every $v \in V$ there exists $u \in U$ with $v \leqslant u$. We let $D_{0}(L)$ be the set of all join-prime elements of $L$, and for $k \in \omega$ we let $D_{k+1}(L)$ be the set of all $a \in L$ such that whenever $a \leqslant \sum U$ for some $U \subseteq L$ and $a \$ u$ for all $u \in U$, then there exists $V \subseteq D_{k}(L)$ such that $V \ll U$ and $a \leqslant \Sigma V$. If $k \leqslant m$, then $D_{k}(L) \subseteq D_{m}(L)$, and we let $D(L)=\cup_{k \in \omega} D_{k}(L)$. Define , $D_{k}^{\prime}(L)$ and $D^{\prime}(L)$ dually. 

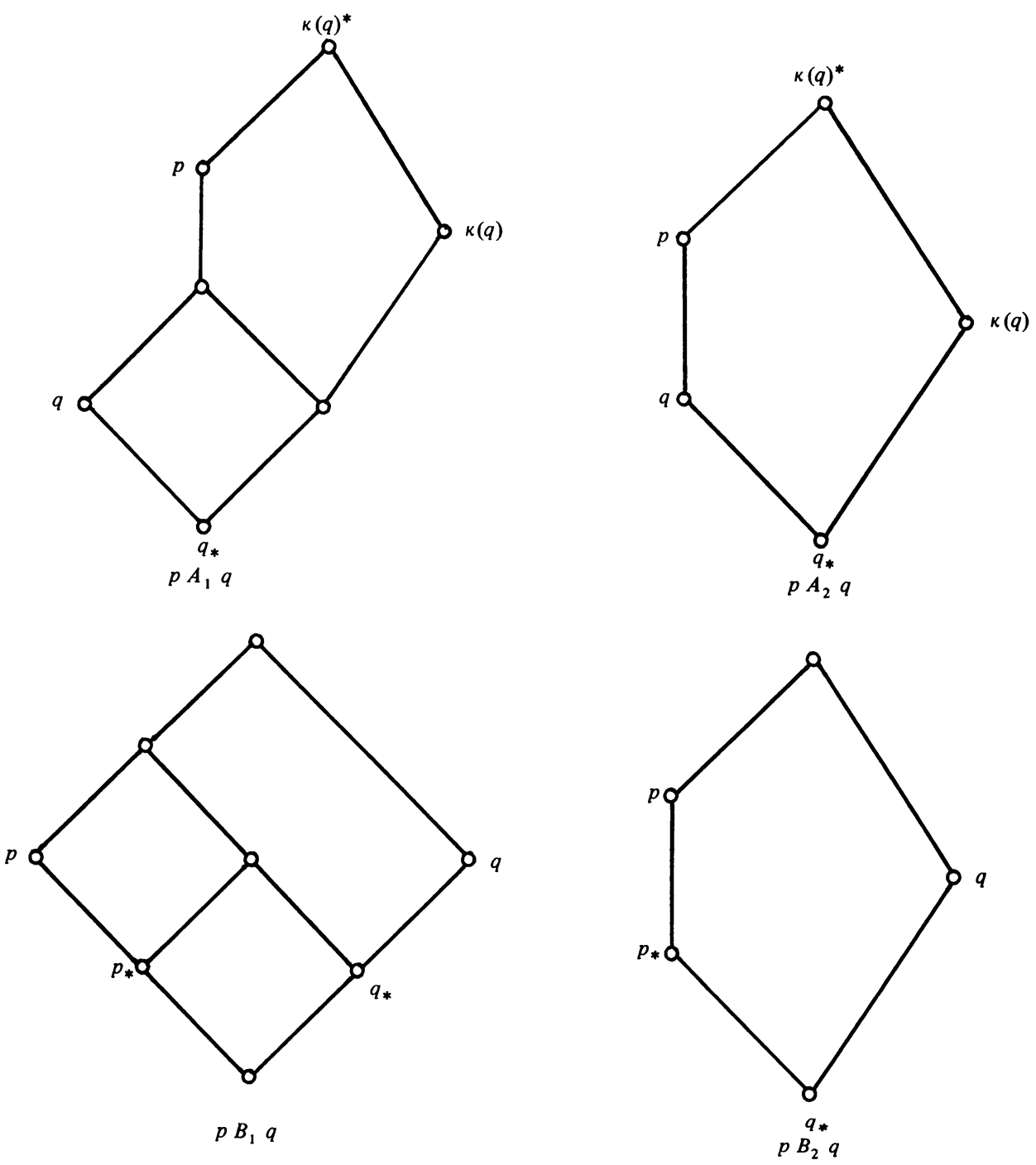

Figure 1

First, we want to show that if a finite lattice $L$ satisfies (W) and $D(L)=L=$ $D^{\prime}(L)$, then $L$ is projective. (The converse is also true; see [6, 9, 15 or 18], and cf. [3].) Let $f: K \rightarrow L$ be a homomorphism. Since $L$ is finite, we can easily find a monotonic transversal $g_{0}: L \succ K$ (i.e., $a \leqslant b$ implies $g_{0}(a) \leqslant g_{0}(b)$, and $f g_{0}(a)=a$ for all $a, b \in L$ ). Inductively we define for all $a \in L$,

$$
g_{k+1}(a)=g_{k}(a) \Pi\left(\sum g_{k}(U): U \subseteq D_{k}(L) \text { and } a<\sum U\right) .
$$

It is then not hard to show that if $a \in D_{k}(L)$ and $m>k$, then $g_{m}(a)=g_{k}(a)$. Since $L$ is finite and we are assuming that $D(L)=L$, we have $D_{n}(L)=L$ for sufficiently large $n$, whence $g_{m}=g_{n}$ for all $m \geqslant n$. Let $h_{0}=g_{n}$, and check that $h_{0}$ is a join-preserving transversal. 
If we dualize the above construction, beginning with $h_{0}$ and using the fact that $D^{\prime}(L)=L$, we obtain a meet-preserving transversal $h$. However, since $h_{0}$ was join-preserving, we can use (W) to show inductively that each $h_{k}(k \geqslant 0)$, and hence $h$, is also join-preserving. Leaving these calculations to the reader, we conclude that $h$ is the desired embedding of $L$ into $K$, and $L$ is projective.

Next we must show that if $L$ is finite, semidistributive and $D(L) \neq L$, then $L$ contains a cycle. First note that if $\varnothing \neq U \subseteq D_{k}(L)$, then $\Sigma U \in D_{k+1}(L)$. Thus if $D(L) \neq L$, some join-irreducible element of $L$ is not in $D(L)$. The existence of a cycle is then a consequence of the following claim and the finiteness of $L$.

If $p \in J(L)-D(L)$, then there exists $q \in J(L)-D(L)$ with $p C q$. For since $p \notin D(L)$, there must exist $U \subseteq L$ such that $p \leqslant \Sigma U$ but $p \$ u$ for all $u \in U$, and for every $V \ll U$ such that $V \subseteq D(L), p \nless \Sigma V$. Since $p \leqslant \sum U$, we have $\Sigma U \nless$ $\kappa(p)$, whence $u_{0} \$ \kappa(p)$ for some $u_{0} \in U$. Choose $y \leqslant u_{0}$ minimal with respect to the property $y \$ \kappa(p)$. Clearly $y \in J(L)$ and $p B y$; if $y \notin D(L)$ we may take $q=y$, and the desired conclusion holds.

Otherwise, $y \in D(L)$ and $p \leqslant p_{*}+y$. Choose a minimal element $z \leqslant p_{*}$ subject to the condition $p \leqslant y+z$. Then $z \notin D(L)$, for otherwise since $z<p \leqslant \Sigma U$, we would have either $z \leqslant u_{1}$ for some $u_{1} \in U$, or else there exists $W \ll U$ such that $W \subseteq D(L)$ and $z \leqslant \Sigma W$. Since also $y \in D(L)$ and $y \leqslant u_{0}$, either case leads to a contradiction. Thus $z \notin D(L)$, and some canonical joinand (see [14]) $q$ of $z$ is not in $D(L)$. Now by Lemma 1.1(ii), the remaining canonical joinands of $z$ (if any) lie below $\kappa(q)$, and by the minimality of $z$ we also have $y \leqslant \kappa(q)$. With this information, it is not hard to check that $p A q$.

From the above arguments we may conclude that if $L$ is an $S$-lattice which is not projective, then either $L$ or $L^{d}$, the dual of $L$, contains a cycle. However, a result of Alan Day [4] (cf. [5, 15, 19]) shows that for a finite semidistributive lattice, $D(L)=L$ iff $D^{\prime}(L)=L$. We will use a more technical version of Day's theorem, from [19], which allows us to transform any cycle into a dual cycle with the roles of $A$ and $B$ interchanged.

LEMMA 1.3. Let $L$ be a finite semidistributive lattice, and $p, q \in J(L)$.

(i) If $p A q$, then $\kappa(p) B^{d} \kappa(q)$.

(ii) If $p B q$, then $\kappa(p) A^{d} \kappa(q)$.

Thus $L$ contains a cycle iff $L^{d}$ does.

Proof. (i) If $p A q$, then $\kappa(p) \neq \kappa(q)$ since $\kappa$ is bijective, and $p \leqslant \kappa(p)^{*} \kappa(q)^{*}$, so $\kappa(p) \ngtr \kappa(p)^{*} \kappa(q)^{*}$. On the other hand, we have $\kappa(p) \geqslant \kappa(p)^{*} \kappa(q)$, for otherwise $\kappa(p)+p=\kappa(p)^{*}=\kappa(p)+\kappa(p)^{*} \kappa(q)$, whence by $\left(\mathrm{SD}_{\vee}\right), \kappa(p)^{*}=\kappa(p)+p \kappa(q)$ $\leqslant \kappa(p)+p_{*}=\kappa(p)$, a contradiction. Thus $\kappa(p) B^{d} \kappa(q)$.

(ii) Let $p B q$. Then $\kappa(p) \geqslant q_{*}$ and $\kappa(p) \neq q$, so $\kappa(p) \leqslant \kappa(q)$. Because $\kappa$ is bijective, $\kappa(p) \neq \kappa(q)$. Thus $\kappa(q)>\kappa(p) \geqslant q_{*}$, as desired.

Combining Lemma 1.3 with the previous arguments, we have proved the direction of Theorem 1.2 which we will be using: if $L$ is an $S$-lattice which is not projective, then $L$ contains a cycle. At this point it would seem appropriate to indicate to the reader our general plan for showing that no $S$-lattice contains a 
cycle. First of all, cycles can exist in finite semidistributive lattices failing (W), e.g., as in Figure 2 (from [15]).

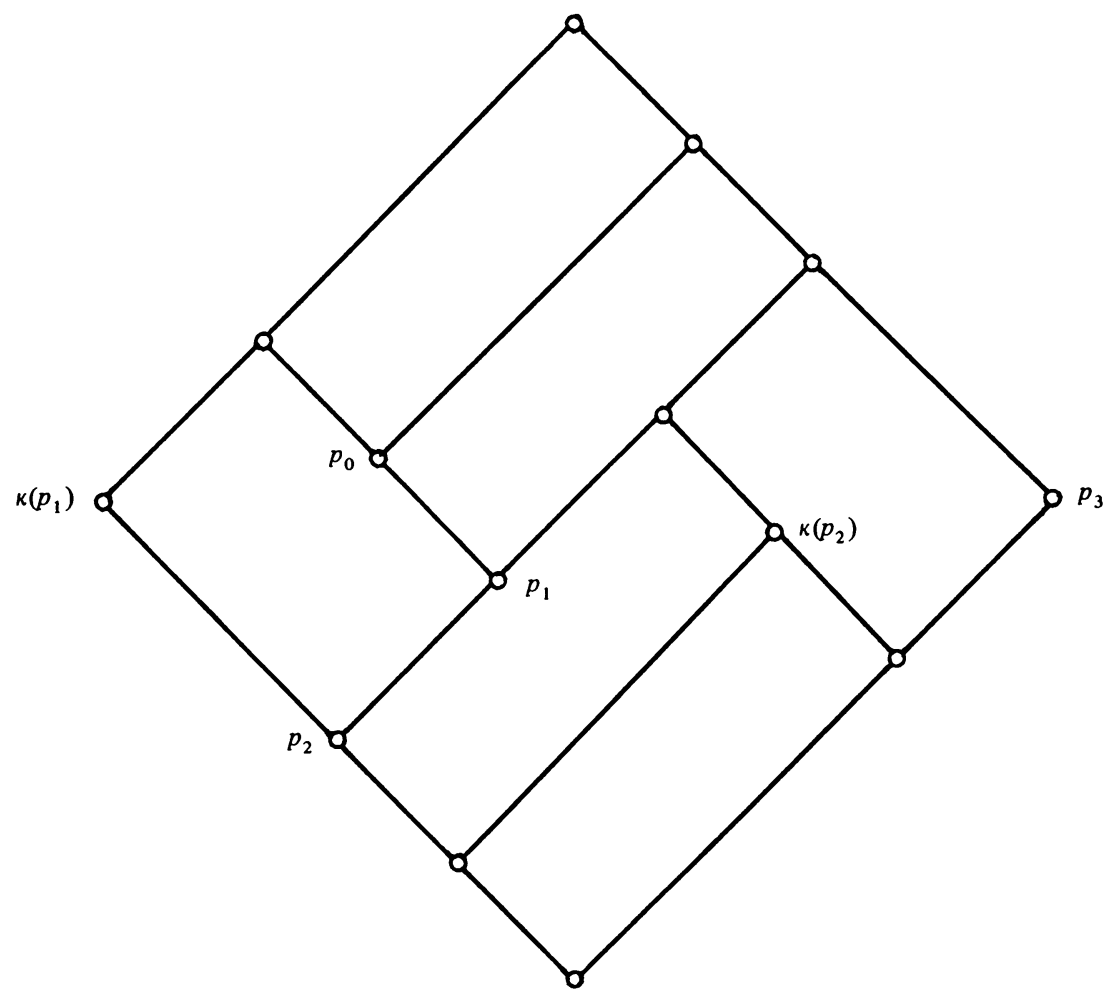

Figure 2. $p_{0} A p_{1} A p_{2} B p_{3} B p_{0}$

However, there are certain configurations which cannot exist in a finite lattice satisfying (W). We will develop two types of these excluded configurations in $\S 2$, and use them repeatedly in the rest of our arguments.

Suppose that $p_{0} C p_{1} C \ldots C p_{n}=p_{0}$ is a minimal cycle in an $S$-lattice. Whenever $p_{i} A p_{i+1}$, then $p_{i}>p_{i+1}$, so of course $p_{i *}>p_{i+1 *}$. Some of our lemmas will state that under the right circumstances, if $p_{i} B p_{i+1} B \ldots B p_{j} A p_{j+1}$, then $p_{i *}>p_{j+1 *}$. Now clearly these circumstances cannot always persist, for then (with appropriate indexing) we could obtain $p_{0 *}>p_{j+1 *}>p_{k+1 *}>\cdots>p_{0 *}$, a contradiction.

Fortunately, however, in those situations where $p_{i} B p_{i+1} B \ldots B p_{j} A p_{j+1}$ and $p_{i *} \ngtr p_{j+1 *}$, one of two things occurs. Observe that for every $p_{i}$ in our minimal cycle, by virtue of $p_{i-1} C p_{i}, p_{i *}$ is meet reducible, while $p_{i} C p_{i+1}$ implies $p_{i *} \neq 0$ (see Figure 1). Therefore $p_{i *} \in J(L)$, and $p_{i * *}$ exists. In most cases, from $p_{i} B p_{i+1} B \ldots B p_{j} A p_{j+1}$ we can conclude that $p_{i *}>p_{j+1 * *}$, whence $p_{i * *} \geqslant$ $p_{j+1 * *}$. In the remaining case, we find that $p_{j} A p_{j+1}$ is a single $A_{2}$ sandwiched 
between $B$ 's, and that this section of the cycle behaves enough like a sequence of all $B$ 's to enable us to use our arguments at the next occurrence of an $A$ in the cycle. (Here we will employ the notion of a $B$-type sequence, which will be defined in \$2.)

Our modified arguments enable us to obtain $p_{0 * *} \geqslant p_{j+1 * *} \geqslant p_{k+1 * *} \geqslant \cdots \geqslant$ $p_{0 * *}$ (with appropriate indexing) for any minimal cycle in an $S$-lattice. Moreover, one of the inequalities will be strict (and thus lead to a contradiction) if our cycle contains any $A_{1}$ or any two consecutive $A$ 's. By the duality induced by Lemma 1.3, neither can our cycle contain two consecutive $B$ 's. Thus the $A$ 's and $B$ 's alternate, in which case we can show that one of the inequalities will be strict if any of the $B$ 's is a $B_{2}$. So we are left only to consider cycles of the form $p_{0} B_{1} p_{1} A_{2} p_{2} B_{1} p_{3} \ldots A_{2} p_{n}=p_{0}$. This type of cycle is excluded by a separate argument, which will complete the proof.

Of course, projectivity and related concepts for lattices have been extensively studied. Several of these ideas which are distinct for general lattices coalesce in the finite case. Combining what is already known with the present result, we obtain the following list of characterizations of finite projective lattices.

THEOREM 1.4. For any finite lattice $L$, the following conditions are equivalent.

(i) $L$ is a sublattice of a free lattice.

(ii) $L$ is projective.

(iii) $L$ is semidistributive and satisfies $(W)$.

(iv) $L$ does not contain any of the lattices $L_{1}-L_{8}$ from Figure 3 as a sublattice.

(v) $L$ is a bounded homomorphic image of a free lattice and satisfies $(W)$.

(vi) $L$ is transferable.

(vii) $L$ is sharply transferable.

The equivalence of (i), (ii) and (v) was found by R. McKenzie [18]; generalizations to infinite lattices were given by R. Freese, B. Jónsson, A. Kostinsky and the author $[6,15,16]$. The equivalence of (iii) and (iv) is due to R. Antonius, B. Davey, W. Poguntke and I. Rival [1, 2]. The equivalence of (vi) and (vii) is due to C. Platt [20], while the equivalence of (i) and (vii) was shown by H. Gaskill, G. Grätzer and C. Platt $[8,9]$ (see also $[10,17,19])$.

Also, two important special cases of Jónsson's conjecture were previously known to be true. I. Rival and B. Sands [21] proved that a planar $S$-lattice is always projective, while J. Ježek and V. Slavik [11] showed that a subdirectly irreducible $S$-lattice is always a sublattice of a free lattice. Ježek and Slavik in fact gave a complete description of all subdirectly irreducible $S$-lattices.

The author would like to thank Bjarni Jónsson for convincing him to pursue this approach to the problem, and Ralph Freese, Tom Harrison and Bill Lampe for their many helpful suggestions and comments.

2. Configuration lemmas. In this section we will develop some configurations which cannot exist in an $S$-lattice, to be used later in showing that no cycle exists. We begin by isolating some useful properties about a string of $B$ 's in a minimal cycle. 

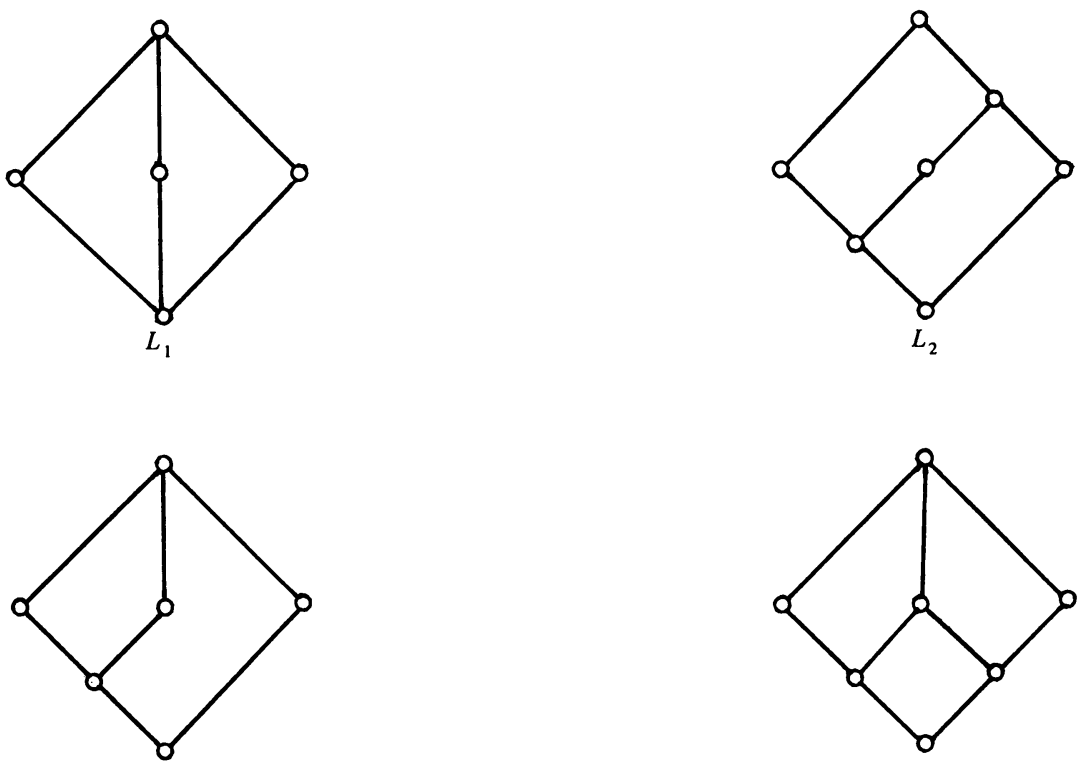

$$
L_{3}=L_{4}^{d}
$$

$$
L_{5}=L_{6}^{d}
$$
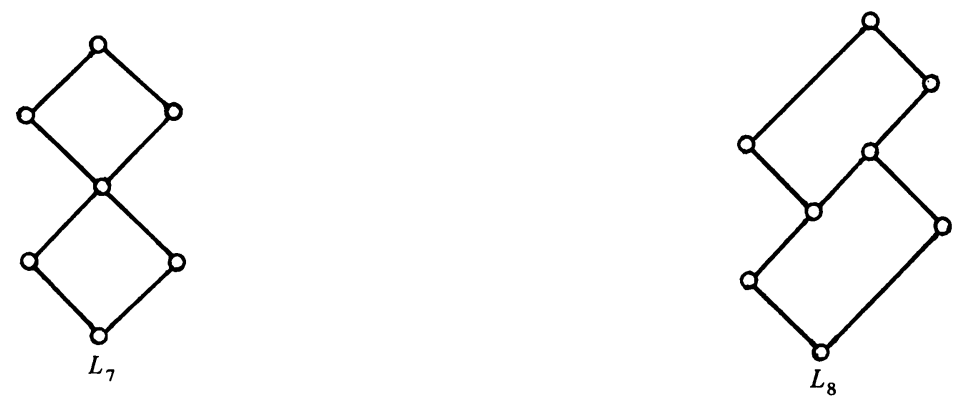

FIGURE 3

LEMMA 2.1. If $\left\langle q_{0}, \ldots, q_{k}\right\rangle$ with $k \geqslant 0$ is a subsequence of a minimal cycle in an $S$-lattice such that $q_{j} B q_{j+1}$ for $0 \leqslant j<k$, then

(i) $q_{j-1 *} \$ q_{j}$ for $1 \leqslant j \leqslant k$,

(ii) $\sum_{i<j} q_{i} \leqslant \kappa\left(q_{j}\right)$ for $1 \leqslant j \leqslant k$,

(iii) $q_{j+1 *} \leqslant \kappa\left(q_{j}\right)$ for $0 \leqslant j \leqslant k-1$,

(iv) $q_{j+i *} \leqslant \kappa\left(q_{j}\right)$ for $i \geqslant 2$ and $0 \leqslant j \leqslant k-i$.

Proof. (i) and (iii) are immediate from the relations $q_{j-1} B q_{j}$ and $q_{j} B q_{j+1}$.

For (ii), recall that $q_{i} B q_{i+1}$ implies $q_{i} \leqslant \kappa\left(q_{i+1}\right)$, and moreover $\kappa\left(q_{i}\right) A^{d} \kappa\left(q_{i+1}\right)$, whence $\kappa\left(q_{i}\right) \leqslant \kappa\left(q_{i+1}\right)$. It follows that, for $i<j, q_{i} \leqslant \kappa\left(q_{i+1}\right) \leqslant \kappa\left(q_{j}\right)$ as claimed.

For (iv), first note that $q_{j+1} \leqslant q_{j+1 *}+q_{j+2}$, and $q_{j+1} \leqslant \kappa\left(q_{j}\right)$ while $q_{j+1 *} \leqslant \kappa\left(q_{j}\right)$, so we must have $q_{j+2} \$ \kappa\left(q_{j}\right)$. If $q_{j+2 *} \leqslant \kappa\left(q_{j}\right)$, we would have $q_{j} B q_{j+2}$, in contradiction to the minimality of our cycle. Thus $q_{j+2 *} \$ \kappa\left(q_{j}\right)$. For $i>2$, we have $q_{j+i *} \leqslant \kappa\left(q_{j+1}\right)$ by induction, and $\kappa\left(q_{j}\right) \leqslant \kappa\left(q_{j+1}\right)$ since $\kappa\left(q_{j}\right) A^{d} \kappa\left(q_{j+1}\right)$, so that $q_{j+i *} \$ \kappa\left(q_{j}\right)$, as desired. 
The proof of (iv) above included the first use of a simple observation which will appear of ten in our arguments. If $p$ and $q$ are distinct elements from a minimal cycle and $q$ is not the successor of $p$ in the cycle, then $p B q$ does not hold; therefore $q \$ \kappa(p)$ implies $q_{*} \$ \kappa(p)$. We shall refer to this argument as the free star principle.

We wish to generalize the situation where $q_{0} B \ldots B q_{k}$. Let $p_{0} C \ldots C p_{n} C p_{0}$ be a minimal cycle in an $S$-lattice, and let $\left\langle q_{0}, \ldots, q_{k}\right\rangle$ be a subsequence of $\left\langle p_{0}, \ldots, p_{n}\right\rangle$ with $k \geqslant 0$. (Thus the $q_{j}$ 's are in their correct order from the cycle, but $q_{j}$ and $q_{j+1}$ need not be consecutive elements in the cycle.) We say that $\left\langle q_{0}, \ldots, q_{k}\right\rangle$ is a $B$-type sequence if conditions (i)-(iv) of Lemma 2.1 are satisfied. The difference between a $B$-type sequence and a sequence of $B$ 's (i.e., $\left.q_{j} B q_{j+1}\right)$ is that we do not require $q_{j+1} \$ \kappa\left(q_{j}\right)$ for a $B$-type sequence. This notion will play a crucial role in our proof.

The first configuration we consider which cannot exist in a finite $S$-lattice comes from [15, Lemma 7.4].

LEMMA 2.2. A finite lattice $L$ satisfying $(W)$ cannot contain elements $a, a_{0}, a_{1}, b, b_{0}$ such that the following conditions hold.

(i) $a$ and $b$ are join-reducible.

(ii) $a \leqslant a_{1} \prec a_{0}$.

(iii) $b \leqslant b_{0}$.

(iv) $a \$ b_{0}$.

(v) $b \$ a_{0}$.

(vi) $a_{0} \$ a_{1}+b_{0}$.

(vii) $b_{0} \$ a_{1}+b$.

A variation of this lemma will also prove useful.

LEMMA 2.3. A finite lattice $L$ satisfying $(W)$ cannot contain elements $a, a_{0}, a_{1}, b, b_{0}$ such that

(i)' $a \in J(L)$ and $a \leqslant a_{*}+b$, and $b$ is join-reducible, and (ii)-(vii) of Lemma 2.2 hold.

These configurations are illustrated in Figure 4.

SKETCH OF PROOFS. Suppose that one of these configurations exists in a finite lattice $L$ satisfying (W). First observe that $a_{1}=a_{0}\left(a_{1}+b_{0}\right)$ is meet-reducible, and hence join-irreducible, since a lattice satisfying $(\mathrm{W})$ contains no doubly reducible elements. We claim that $a<a_{1}$. In Lemma 2.2, this follows because $a$ is joinreducible, while $a_{1} \in J(L)$. In Lemma 2.3 , we have $a_{1}=a_{0}\left(a_{1}+b_{0}\right) \nless a_{1 *}+b$ by (W), while $a \leqslant a_{*}+b$, so that again $a \neq a_{1}$.

Let $a_{2}=a_{1 *}$ and $b_{1}=b_{0}\left(a_{1}+b\right)$. The reader can now check that (i)-(vii) hold with $a_{0}, a_{1}, b_{0}$ replaced by $a_{1}, a_{2}, b_{1}$. Therefore by iterating this process we can obtain two infinite descending chains, $\left\{a_{i}: i \in \omega\right\}$ and $\left\{b_{j}: j \in \omega\right\}$, contrary to the finiteness of $L$. It follows that the configurations cannot exist.

The configurations of Lemmas 2.2 and 2.3 can arise very naturally when we consider sequences of the type $q_{0} B \ldots B q_{k} A p$. Thus our most frequent applications of Lemma 2.3 will be in the form of the following lemma, or some variation thereof. 


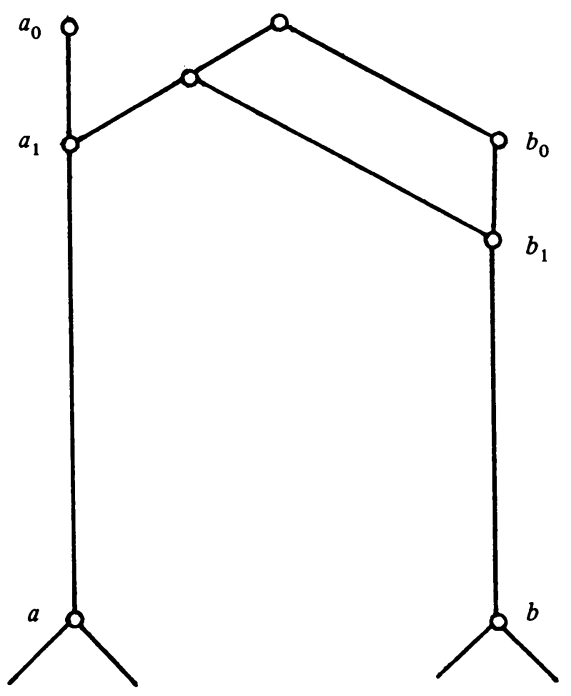

LEMMA 2.2

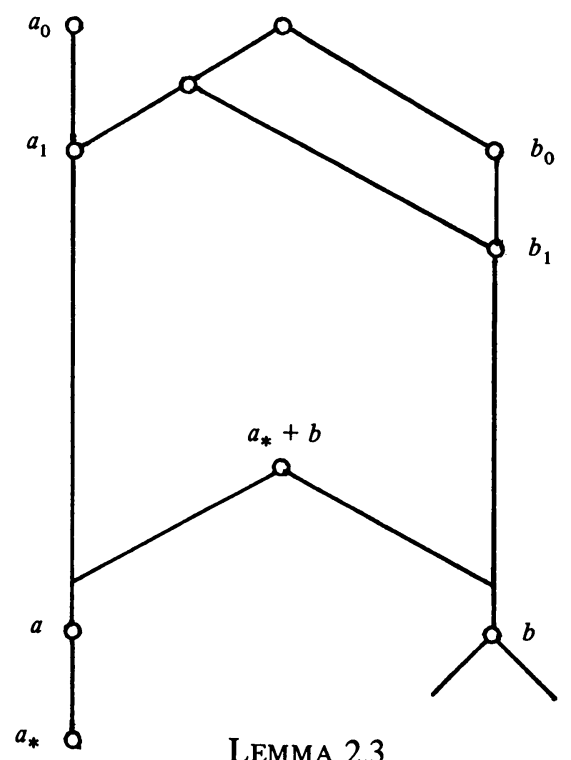

LEMMA 2.3

FIGURE 4

LEMMA 2.4. Let $\left\langle q_{0}, \ldots, q_{k}, p\right\rangle$ with $k \geqslant 1$ be a subsequence of a minimal cycle in an $S$-lattice, and assume that

(i) $\left\langle q_{0}, \ldots, q_{k}\right\rangle$ is a $B$-type sequence.

(ii) $q_{k} A_{2} p$.

(iii) $p_{* *} \$ q_{0 *}$.

(iv) $p B q_{0}$ does not hold.

Let $j>1$ be chosen minimal with respect to the property $p_{*} \leqslant q_{j *}$. Then $\Sigma_{i<j} q_{i}<$ $\kappa(p)$.

Proof. First of all, observe that $p_{*} \nless q_{0 *}$ by (iii), while $p_{*}<q_{k *}$ by (ii). Therefore $j$ can be chosen as indicated. Supposing that $\Sigma_{i<j} q_{i} \$ \kappa(p)$, by the free star principle and (iv) we also have $\Sigma_{i<j} q_{i *} \$ \kappa(p)$. Let us apply Lemma 2.3.

We must choose $a, a_{0}, a_{1}, b, b_{0}$. Let

$$
\begin{aligned}
& a=p, \quad b=p_{* *}+\sum_{i<j} q_{i *}, \\
& b_{0}=p_{* *}+\sum_{i<j-1} q_{i *}+q_{j-1} .
\end{aligned}
$$

If $j=k$, let $a_{0}=q_{k}$ and $a_{1}=q_{k *}$. Otherwise $j<k$, and we choose $a_{0}$ and $a_{1}$ as follows. Note that $k-(j-1) \geqslant 2$, so that by property $2.1(\mathrm{iv}), q_{k *} \nless \kappa\left(q_{j-1}\right)$. On the other hand, $p_{*} \leqslant q_{j *} \leqslant \kappa\left(q_{j-1}\right)$ by property 2.1 (iii), whence by the free star principle $p \leqslant \kappa\left(q_{j-1}\right)$. Pick $a_{0}$ minimal in the interval $\left[p, q_{k *}\right]$ with respect to the property $a_{0} \$ \kappa\left(q_{j-1}\right)$. Since $a_{0}$ is clearly join-irreducible in the interval [ $\left.p, q_{k *}\right], a_{0}$ covers a unique element $a_{1}$ in the interval. Note that in either case, whether $j=k$ or $j<k$, we have $a_{1} \leqslant \kappa\left(q_{j-1}\right)$. (If $j<k$, this is clear; if $j=k$, use property 2.1(iii).) 
Now let us check that the seven conditions of Lemma 2.3 hold.

(i) $a=p \in J(L)$, and since $\Sigma_{i<j} q_{i *} \$ \kappa(p)$, we have $p \leqslant p_{*}+\Sigma_{i<j} q_{i *}=p_{*}$ $+b$. To see that $b$ is join-reducible, we show that $b \neq p_{* *}$ and $b \neq q_{i *}$ for $i<j$. Now $b \neq p_{* *}$, since $q_{j-1 *} \leqslant b$ while $q_{j-1 *} \$ p_{* *}$, as $p_{* *}<q_{j}$ and, by 2.1(i), $q_{j-1 *} \$ q_{j}$. Also $b \neq q_{0 *}$, since $p_{* *} \leqslant b$ while $p_{* *} \$ q_{0 *}$. If $j=1$, we are done. Otherwise, for $1 \leqslant i<j$ we have $q_{i-1 *} \leqslant b$ while $q_{i-1 *} \leqslant q_{i}$ by $2.1(\mathrm{i})$, wherefore $b \neq q_{i *}$. Thus $b$ is join-reducible.

Conditions (ii) and (iii), $a \leqslant a_{1} \prec a_{0}$ and $b \leqslant b_{0}$, are immediate.

(iv) If $a \leqslant b_{0}$, then using $q_{k} A_{2} p$ we have $q_{k} \kappa(p)=p_{*}=a_{*} \leqslant b_{0}$, so we may apply (W) to the inclusion

$$
q_{k} \kappa(p) \leqslant p_{* *}+\sum_{i<j-1} q_{i *}+q_{j-1} .
$$

Now $q_{k} \$ b_{0}$ (the right-hand side) since by 2.1 (ii), $b_{0} \leqslant \kappa\left(q_{k}\right)$. Also $\kappa(p) \$ b_{0}$, for since $q_{k} A p$ we have $q_{k} \leqslant \kappa(p)^{*}=p+\kappa(p)$, while $p+b_{0} \leqslant \kappa\left(q_{k}\right)$ as before. Of course $q \kappa(p)=p_{*} \$ p_{* *}$, and $p_{*} \$ q_{i *}$ for $0 \leqslant i \leqslant j-1$ by the choice of $j$. This leaves only the possibility $p_{*}=q_{j-1}$, which however would imply $q_{j-1 *}<p_{*}<q_{j}$, contrary to 2.1(i). Therefore $a \nless b_{0}$.

(v) If $b \leqslant a_{0}$, then $q_{j-1 *} \leqslant b \leqslant a_{0} \leqslant q_{k}$. If $j=k$, this contradicts 2.1(i), so we may assume $j<k$. Now $q_{j-1 *} \$ \kappa(p)$, or else we would have $q_{j-1 *} \leqslant q_{k} \kappa(p)=p_{*}$ $\leqslant q_{j}$, contrary to 2.1(i). Therefore $\kappa(p)^{*}=p+\kappa(p)=q_{j-1 *}+\kappa(p)$, since $p \leqslant p_{*}$ $+q_{j-1 *} \leqslant q_{j-1 *}+\kappa(p)$ and $q_{j-1 *} \leqslant q_{k} \leqslant \kappa(p)^{*}$. Applying $\left(\mathrm{SD}_{\vee}\right)$ yields $\kappa(p)^{*}=$ $p q_{j-1 *}+\kappa(p)$. Since $p_{*} \leqslant \kappa(p)$, however, we cannot have $p q_{j-1 *}<p$; therefore $p \leqslant q_{j-1 *}$. If $j=1$, this contradicts one of our original assumptions; otherwise we continue. Now $q_{j-1 *}<q_{k}$, because $p_{*} \leqslant q_{k}$ but $p_{*} \$ q_{j-1 *}$. Also, since $j<k$, we have $k-(j-1) \geqslant 2$, whence by 2.1 (iv), $q_{j-1} \leqslant q_{j-1 *}+q_{k *}=q_{k *}$. Thus $p<q_{j-1}$ $<q_{k} \leqslant \kappa(p)^{*}$, and $q_{j-1} A p$. This, however, contradicts the minimality of our original cycle. We conclude that $b \$ a_{0}$.

(vi) If $a_{0} \leqslant a_{1}+b_{0}$, then $j \neq k$ (i.e., $a_{0} \neq q_{k}$ ), for by 2.1(ii), $b_{0} \leqslant \kappa\left(q_{k}\right)$. Thus $j<k$, so that $a_{0} \$ \kappa\left(q_{j-1}\right)$ by the choice of $a_{0}$, whence $q_{j-1} \leqslant q_{j-1 *}+a_{0}$. Since also $a_{0} \leqslant a_{1}+b_{0}$ and $p_{* *}<p_{*}=a$, we compute

$$
a_{0}+b_{0}=a_{0}+\sum_{i<j} q_{i *}=a_{1}+\sum_{i<j-1} q_{i *}+q_{j-1} .
$$

Applying $\left(\mathrm{SD}_{\vee}\right)$ in its more general form, we obtain $a_{0}+b_{0}=a_{1}+\sum_{i<j} q_{i *}+$ $a_{0} q_{j-1}$. However, in (v) we showed that $q_{j-1 *} \$ q_{k}$, so $q_{j-1} \$ a_{0}$. Hence $a_{0} q_{j-1} \leqslant$ $q_{j-1 *}$, and

$$
a_{0}+b_{0}=a_{1}+\sum_{i<j} q_{i *}
$$

But $a_{1}+\sum_{i<j} q_{i *} \leqslant \kappa\left(q_{j-1}\right)$ by the choice of $a_{0}$ and 2.1 (ii), while $q_{j-1} \leqslant b_{0} \leqslant a_{0}+$ $b_{0}$, so this is a contradiction. Thus $a_{0} \$ a_{1}+b_{0}$.

(vii) Finally $b_{0} \leqslant a_{1}+b$, else $q_{j-1} \leqslant b_{0} \leqslant a_{1}+b=a_{1}+\sum_{i<j} q_{i *} \leqslant \kappa\left(q_{j-1}\right)$ as above, a contradiction.

By Lemma 2.3 then, this configuration cannot occur, and we conclude that $\sum_{i<j} q_{i} \leqslant \kappa(p)$. 
The second type of configuration we wish to consider which does not occur in a finite lattice satisfying (W) is also found in [15, Lemma 7.2]. It was inspired by $P$. Whitman's result [24] that a subset $X$ of a lattice satisfying (W) generates a free lattice iff $a \nless \sum F$ and $a \ngtr \Pi F$ whenever $a \in X$ and $F$ is a finite subset of $X$ with $a \notin F$ (cf. Jónsson [13]).

LEMMA 2.5. A finite lattice $L$ satisfying ( $W$ ) cannot contain elements $a, b, c$ such that the following conditions hold.

(i) $b(c+a b) \$ a$.

(ii) $a(c+a b) \$ b$.

(iii) $a b \$ c$.

(iv) $a \$ b+c$.

(v) $b \$ a+c$.

SKETCH OF PROOF. Supposing that (i)-(v) hold, let $a_{1}=a(b+c)$ and $b_{1}=$ $b(a+c)$. Then $a_{1}<a$ and $b_{1}<b$, and it is fairly simple to show that (i)-(v) hold with $a$ and $b$ replaced by $a_{1}$ and $b_{1}$. Thus we obtain two infinite descending chains, contrary to the finiteness of $L$.

We will most often use, instead of Lemma 2.5, the following simplified, dualized, and disguised version of this configuration (cf. H. Rolf [22]).

LEMMA 2.6. A finite lattice $L$ satisfying $(W)$ cannot contain elements $a, a_{0}, b, b_{0}$ such that the following conditions hold.

(i) $a \leqslant a_{0}$.

(ii) $b \leqslant b_{0}$.

(iii) $a \$ b_{0}$.

(iv) $b \$ a_{0}$.

(v) $a_{0} \$ a+b$.

(vi) $b_{0} \$ a+b$.

(vii) $a_{0} b \$ a$.

(viii) $a b_{0} \$ b$.

Proof. Supposing that (i)-(viii) hold, let $c=a_{0} b_{0}$. Then $a \$ b+c$ since $b+c$ $\leqslant b_{0}$, and similarly $b \leqslant a+c$. By (W), $c \leqslant a+b$; for (v) and (vi) say that $a_{0}$, $b_{0} \$ a+b$, while $a_{0} b \leqslant c$ and $a b_{0} \leqslant c$ imply $c \$ a, b$ using (vii) and (viii). On the other hand, conditions (vii) and (viii) say directly that $b c \nless a$ and $a c \$ b$. The five noninclusions we have just shown are stronger than the duals of the conditions of Lemma 2.5, and we conclude that the configuration cannot occur in $L$.

(Conversely, if $a, b, c$ satisfy $a \$ b+c, b \$ a+c, c \$ a+b, b c \$ a$ and $a c \$ b$, we may let $a_{0}=a+c$ and $b_{0}=b+c$. It is straightforward to check that 2.6(i)-(viii) hold.)

Now it turns out that the configuration of Lemma 2.6 also tends to arise when we consider sequences of the form $q_{0} B \ldots B q_{k} A p$. All of our applications of Lemma 2.6 are included in the following lemma. 
LEMMA 2.7. Let $\left\langle q_{0}, \ldots, q_{k}, p\right\rangle$ with $k \geqslant 1$ be a subsequence of a minimal cycle in an $S$-lattice $L$, and let $j$ be fixed with $1 \leqslant j \leqslant k$. Assume that for some $t \in L$ the following conditions are satisfied.

(i) $\left\langle q_{0}, \ldots, q_{k}\right\rangle$ is a $B$-type sequence.

(ii) $p_{*} \$ q_{0 *}$.

(iii) $p_{*} \leqslant q_{j *}$

(iv) $p<t \leqslant \kappa(p)^{*}$.

(v) $t \leqslant \kappa\left(q_{j-1}\right)$.

(vi) $t \$ p+\sum_{i<j-1} q_{i *}+q_{j-1}$.

(vii) $p \quad B \quad q_{0}$ does not hold.

Then $\sum_{i<j} q_{i *} \$ \kappa(p)$.

Proof. Suppose that $\Sigma_{i<j} q_{i *} \leqslant \kappa(p)$, whence by the free star principle and (vii) we also have $\Sigma_{i<j} q_{i} \leqslant \kappa(p)$. Let us apply Lemma 2.6 with

$$
\begin{aligned}
& a=p+\sum_{i<j} q_{i *}, \quad a_{0}=t+\sum_{i<j} q_{i *}, \\
& b=p_{*}+\sum_{i<j-1} q_{i *}+q_{j-1}, \quad b_{0}=\kappa(p) .
\end{aligned}
$$

We need to check conditions (i)-(viii) of Lemma 2.6.

Conditions (i) $-(\mathrm{v})$ are easy. That $a \leqslant a_{0}$ and $b \leqslant b_{0}$ are consequences of our assumptions, and $a \$ b_{0}$ is clear. By hypothesis (v) and 2.1(ii), we have $a_{0} \leqslant$ $\kappa\left(q_{j-1}\right)$, whereas $q_{j-1} \leqslant b$, from which $a_{0} \$ b$ follows. By hypothesis (vi), $t \$ a+b$ $=p+\sum_{i<j-1} q_{i *}+q_{j-1}$, which yields $a_{0} \$ a+b$.

For (vi), suppose $b_{0} \leqslant a+b$. Then we would have $t \leqslant \kappa(p)^{*}=p+\kappa(p) \leqslant a+$ $b$, contrary to hypothesis (vi) again. Thus $b_{0} \$ a+b$.

(vii) If $a_{0} b \leqslant a$, then we can apply (W) to the inclusion

$$
\left[t+\sum_{i<j} q_{i *}\right]\left[p_{*}+\sum_{i<j-1} q_{i *}+q_{j-1}\right] \leqslant p+\sum_{i<j} q_{i *}
$$

Now $t \$ a$ (the right-hand side) as $t \$ a+b$. Since $p<t \leqslant \kappa\left(q_{j-1}\right)$ and $\Sigma_{i<j} q_{i *}$ $\leqslant \kappa\left(q_{j-1}\right)$ by $2.1(\mathrm{ii})$, we have $a \leqslant \kappa\left(q_{j-1}\right)$. Hence the second term is not below $a$. On the other hand, we cannot have $a_{0} b \leqslant p$, for that would imply $a_{0} b \leqslant p \kappa(p)=p_{*}$, and thence $q_{j-1 *} \leqslant a_{0} b \leqslant p_{*} \leqslant q_{j}$, contrary to 2.1(i). Since $p_{*} \leqslant a_{0} b$ and $p_{*} \leqslant q_{0 *}$, we cannot have $a_{0} b \leqslant q_{0 *}$. For $1 \leqslant i<j$, though, $q_{i-1 *} \leqslant a_{0} b$ implies $a_{0} b \leqslant q_{i *}$ by 2.1(i). Therefore $a_{0} b \$ a$.

(viii) If $a b_{0} \leqslant b$, then we may apply (W) to the inclusion

$$
\left[p+\sum_{i<j} q_{i *}\right] \kappa(p) \leqslant p_{*}+\sum_{i<j-1} q_{i *}+q_{j-1} .
$$

Now $p$ is not below the right-hand side, $b$, since $b \leqslant \kappa(p)$. If $\kappa(p) \leqslant b$, then $b_{0}=\kappa(p) \leqslant b \leqslant a+b$, contrary to condition (vi) which was proved above. On the other hand, $q_{j-1 *} \leqslant a b_{0}$ implies $a b_{0} \leqslant p_{*}$ since $p_{*} \leqslant q_{j}$. Because $p_{*} \leqslant a b_{0}$ and $p_{*} \$ q_{0 *}$, we have $a b_{0} \$ q_{0 *}$. If $j=1$, we must also note that $a b_{0} \neq q_{0}$ since in this case $a b_{0} \leqslant a \leqslant \kappa\left(q_{j-1}\right)=\kappa\left(q_{0}\right)$. Otherwise, for $1 \leqslant i \leqslant j-1, q_{i-1 *} \leqslant a b_{0}$ implies $a b_{0} \$ q_{i}$ by 2.1(i); a fortiori $a b_{0} \$ q_{i *}$ for $1 \leqslant i<j-1$. Therefore $a b_{0} \$ b$. 
By Lemma 2.6 then, this configuration cannot exist in $L$, and we conclude that $\Sigma_{i<j} q_{i *} \$ \kappa(p)$.

3. Major lemmas. In the preceding section we developed two types of configuration lemmas, and showed how each can be applied to a situation where $\left\langle q_{0}, \ldots, q_{k}\right\rangle$ is a $B$-type sequence and $q_{k} A p$. What is nice about Lemmas 2.4 and 2.7 is that, with rather similar hypotheses, they yield opposite conclusions. (Note that the argument of the former gives rise to infinite descending chains, while that of the latter yields infinite ascending chains.) Thus, in situations where neither one of our configurations gives the desired conclusion, we can try to play the two against one another to reach a contradiction.

In this section we will use the configurations to show that, in every situation where $\left\langle q_{0}, \ldots, q_{k}\right\rangle$ is a $B$-type sequence and $q_{k} A p$, either $q_{0 *}>p_{* *}$ or $\left\langle q_{0}, \ldots, q_{k}\right\rangle$ can be replaced by a longer $B$-type sequence (starting at $q_{0}$ and ending beyond $q_{k}$ in our minimal cycle).

Lemma 3.1. If $\left\langle q_{0}, \ldots, q_{k}\right\rangle$ with $k \geqslant 0$ is a $B$-type sequence and $q_{k} A_{1} p$, then $q_{0 *}>p_{*}$.

Proof. We will proceed by induction on $k$. The case $k=0$ is trivial, for then $q_{0} A p$ implies $q_{0}>p$, whence $q_{0 *}>p_{*}$. (The case $k=1$ may also be found as Lemma 7.5 of [15].)

If $k>0$, then $\left\langle q_{1}, \ldots, q_{k}\right\rangle$ is also a $B$-type sequence. Therefore, by the inductive hypothesis, we may assume that $q_{1 *}>p_{*}$. Hence it follows that $q_{0 *} \nless p_{*}$, for else we would have $q_{0 *} \leqslant p_{*}<q_{1}$, contrary to 2.1(i).

Suppose $q_{0 *} \ngtr p_{*}$. By the preceding remark, we then have in fact $q_{0 *} \ngtr p_{*}$. We will apply Lemma 2.2, mimicking the argument of Lemma 2.4.

We must choose $a, a_{0}, a_{1}, b, b_{0}$. Now $q_{k} \kappa(p) \leqslant q_{k *}$ as $p<q_{k}$ implies $q_{k} \$ \kappa(p)$, and $q_{k} \kappa(p) \$ q_{0 *}$ since $p_{*} \leqslant q_{k} \kappa(p)$. Therefore we may find $j \geqslant 1$ minimal with respect to the property $q_{k} \kappa(p) \leqslant q_{j *}$. Let $a=p+q_{k} \kappa(p), b=p_{*}+\Sigma_{i<j} q_{i *}$, and $b_{0}=p_{*}+\sum_{i<j-1} q_{i *}+q_{j-1}$. If $j=k$, let $a_{0}=q_{k}$ and $a_{1}=q_{k *}$. Otherwise we choose $a_{0}$ and $a_{1}$ as follows. Since $k-(j-1) \geqslant 2$ if $j<k$, by 2.1(iv) we have $q_{k *} \leqslant \kappa\left(q_{j-1}\right)$. On the other hand, $p_{*} \leqslant q_{k} \kappa(p) \leqslant q_{j *} \leqslant \kappa\left(q_{j-1}\right)$ by 2.1(iii), whence by the free star principle $p \leqslant \kappa\left(q_{j-1}\right)$. Thus also $p+q_{k} \kappa(p) \leqslant \kappa\left(q_{j-1}\right)$. Pick $a_{0}$ minimal in the interval $\left[p+q_{k} \kappa(p), q_{k *}\right]$ with respect to the property $a_{0} \leqslant \kappa\left(q_{j-1}\right)$. Clearly $a_{0}$ is join-irreducible in the interval [ $p+q_{k} \kappa(p), q_{k *}$, so $a_{0}$ covers a unique element $a_{1}$ in the interval. Note that in either case, whether $j=k$ or $j<k$, we have $a_{1} \leqslant \kappa\left(q_{j-1}\right)$.

Now we must check that the seven conditions of Lemma 2.2 hold. Except for condition (i), this is done exactly as in the proof of Lemma 2.4, but using $q_{k} \kappa(p)$ in place of $p_{*}$, and $p_{*}$ instead of $p_{* *}$. (Where before we had $p_{* *}<q_{k} \kappa(p)=p_{*}$ and $p_{* *} \$ q_{0 *}$, we now have $p_{*}<q_{k} \kappa(p)$ and $p_{*} \$ q_{0 *}$.) This task will be left to the reader.

For condition (i), we have immediately that $a=p+q_{k} \kappa(p)$ is join-reducible because $q_{k} A_{1} p$, i.e., $q_{k} \kappa(p) \$ p$ (see Figure 1). The argument that $b$ is join-reducible is adapted as above from that given in the proof of Lemma 2.4 . 
By Lemma 2.2, we conclude that this configuration cannot exist in $L$, and hence $q_{0 *}>p_{*}$.

Next, we must start considering what happens when $q_{k} A_{2} p$. First, let us recall Lemma 7.1 from [15].

LEMMA 3.2. If $p_{0} A_{2} p_{1} A p_{2}$ in a minimal cycle, then $p_{1 *}=p_{2}$ and $p_{1} A_{2} p_{2}$.

SKetch OF PROOF. Apply (W) to the inclusion $p_{0} \kappa\left(p_{1}\right)=p_{1 *} \leqslant p_{2}+\kappa\left(p_{2}\right)$.

It is also true that a minimal cycle cannot contain more than two $A_{2}$ 's consecutively (see the proof of Lemma 8.5 of [15]), but we will not use this fact. Indeed, the following lemma shows that, for our purposes, two $A_{2}$ 's behave essentially like an $A_{1}$.

LEMMA 3.3. If $\left\langle q_{0}, \ldots, q_{k}\right\rangle$ with $k \geqslant 0$ is a $B$-type sequence and $q_{k} A_{2} p_{1} A_{2} p_{2}$, then $q_{0 *}>p_{2 *}$.

Proof. Again we proceed by induction, with the case $k=0$ following trivially from $q_{0} A p_{1} A p_{2}$. Thus we may assume $k>0$ and $q_{1 *}>p_{2 *}$.

Suppose $q_{0 *} \ngtr p_{2 *}$. Now $q_{0 *} \$ p_{2 *}$ since $p_{2 *}<q_{1}$; hence we have $q_{0 *} \ngtr p_{2 *}$. (Note that this implies $p_{2} \neq q_{0}$.) Observe that by Lemma 3.2, $p_{1 * *}=p_{2 *}$. So choosing $j$ minimal with respect to the property $p_{2}\left(=p_{1 *}\right) \leqslant q_{j *}$, let us apply Lemma 2.4 with $p=p_{1}$. It is easy to verify that the hypotheses of Lemma 2.4 hold, and we conclude that $\Sigma_{i<j} q_{i} \leqslant \kappa\left(p_{1}\right)$.

Now, however, we are in a position to apply Lemma 2.7 with $p=p_{2}$ and $t=p_{1}$. Condition 2.7(i)-(iv) are easy to check.

For (v), note that $p_{1 *}=p_{2} \leqslant q_{j *} \leqslant \kappa\left(q_{j-1}\right)$ by 2.1 (iii). Thus by the free star principle $p_{1}=t \leqslant \kappa\left(q_{j-1}\right)$.

From our application of Lemma 2.4 above, we have $p_{1} \nless p_{2}+\Sigma_{i<j} q_{i}$, from which (vi) follows immediately.

If (vii) failed, i.e., $p_{2} B q_{0}$, then we could apply (W) to the inclusion

$$
q_{k} \kappa\left(p_{1}\right)=p_{2} \leqslant p_{2 *}+q_{0}
$$

Of course, $q_{k} \nless p_{2 *}+q_{0}$ since by 2.1 (ii), $p_{2 *}+q_{0} \leqslant \kappa\left(q_{k}\right)$. Likewise $\kappa\left(p_{1}\right) \nless p_{2 *}+$ $q_{0}$, for otherwise $q_{k} \leqslant \kappa\left(p_{1}\right)^{*}=p_{1}+\kappa\left(p_{1}\right) \leqslant p_{1}+q_{0} \leqslant \kappa\left(q_{k}\right)$, a contradiction. Surely $p_{2} \$ p_{2 *}$, and $p_{2} \$ q_{0}$ since $p_{2 *} \$ q_{0 *}$. Thus (vii) holds.

Lemma 2.7 then yields $\sum_{i<j} q_{i *} \$ \kappa\left(p_{2}\right)$. That being the case, we may apply (W) to the inclusion

$$
q_{k} \kappa\left(p_{1}\right)=p_{2} \leqslant p_{2 *}+\sum_{i<j} q_{i *}
$$

However, $q_{k}$ is not below the right-hand side since $p_{2 *}+\sum_{i<j} q_{i *} \leqslant \kappa\left(q_{k}\right)$ by 2.1(ii). Similarly $\kappa\left(p_{1}\right)$ is not below the right-hand side, for otherwise $q_{k}<\kappa\left(p_{1}\right)^{*}=$ $p_{1}+\kappa\left(p_{1}\right) \leqslant p_{1}+\sum_{i<j} q_{i *} \leqslant \kappa\left(q_{k}\right)$, a contradiction. Of course $p_{2} \nless p_{2 *}$, while $p_{2} \$ q_{i *}$ for $i<j$ by the choice of $j$.

Since this (W)-failure cannot occur, it must be that our original assumption $q_{0 *} \ngtr p_{2 *}$ was wrong, as desired. 
So it remains for us to consider what happens when $q_{k} A_{2} p B r$. One case of this situation is reasonably easy, so let us do it first.

LemMa 3.4. Let $\left\langle q_{0}, \ldots, q_{k}\right\rangle$ with $k \geqslant 0$ be a $B$-type sequence, and let $q_{k} A_{2} p$. If $p B q_{0}$ does not hold and $q_{0} \$ \kappa(p)$, then $q_{0 *}>p_{* *}$.

Proof. The case $k=0$ is trivial, so we may assume $k \geqslant 1$. Suppose $q_{0 *} \ngtr p_{* *}$, and let us apply Lemma 2.4 .

To verify the hypotheses of that lemma, we need only show that $q_{0 *} \neq p_{* *}$, the rest being immediate. Since $q_{0} \$ \kappa(p)$ and $p B q_{0}$ does not hold, we have $q_{0 *} \$$ $\kappa(p)$, whence $q_{0 *} \neq p_{* *}$.

We conclude from Lemma 2.4 that $\Sigma_{i<j} q_{i} \leqslant \kappa(p)$, which contradicts our hypothesis that $q_{0} \$ \kappa(p)$. Therefore $q_{0 *}>p_{* *}$.

Now we must deal with the case $q_{k} A_{2} p B r$ with $q_{0} \leqslant \kappa(p)$, where it will not be possible to conclude that $q_{0 *}>p_{* *}$ (see Figure 5). Most of our effort will be involved in showing that we can obtain a longer $B$-type sequence when this fails to occur. Our next three lemmas provide the preliminaries for Lemma 3.8.

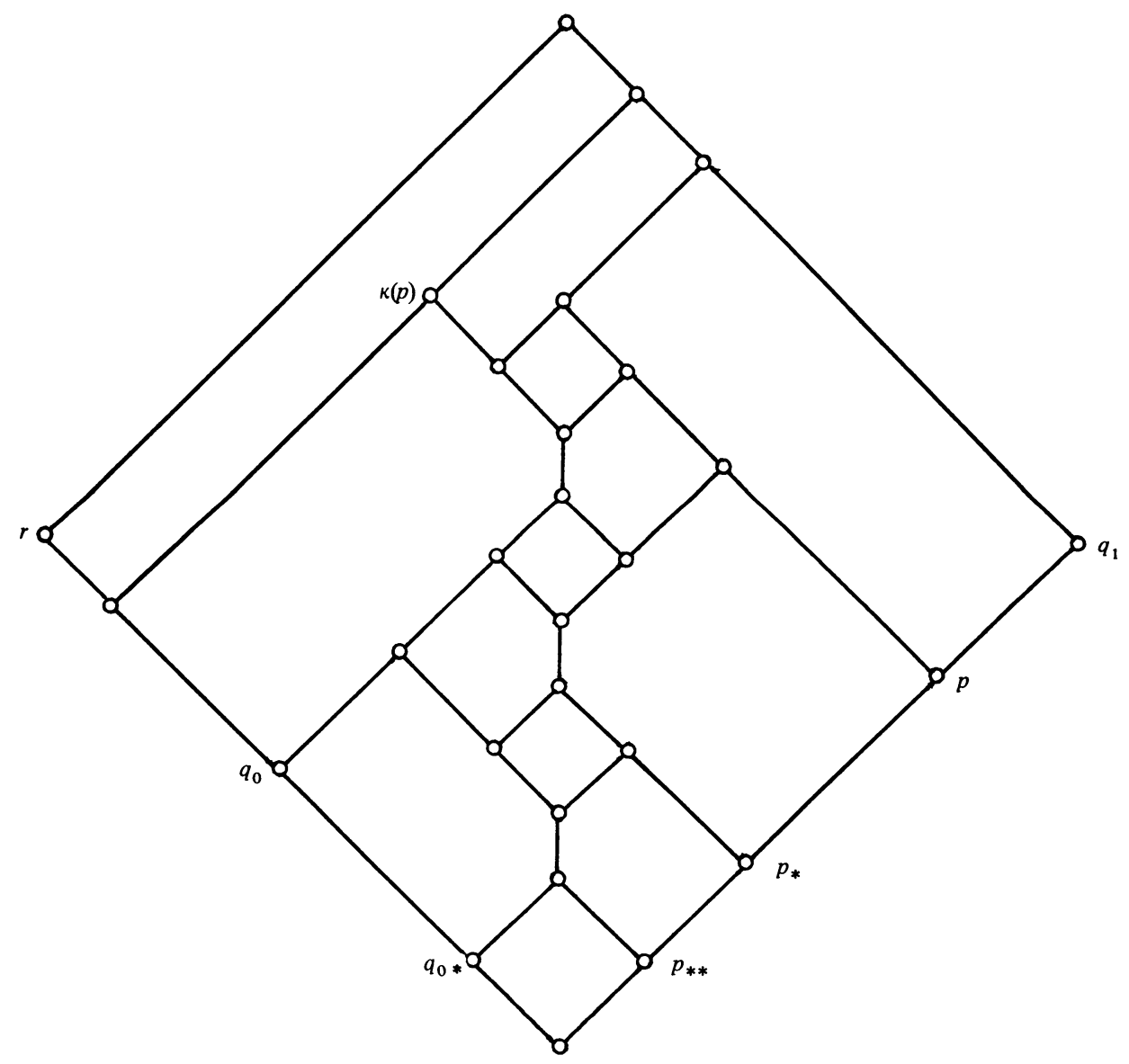

Figure 5 
LeMma 3.5. Let $\left\langle q_{0}, \ldots, q_{k}\right\rangle$ with $k>1$ be a B-type sequence, and let $q_{k} A_{2} p$. If $q_{0} \leqslant \kappa(p)$ and $q_{0 *} \ngtr p_{*}$, then $\Sigma_{i<k} q_{i} \leqslant \kappa(p)$.

Proof. First of all, we note that in this situation $q_{0 *} \neq p_{*}$, so that in fact we have $q_{0 *} \ngtr p_{*}$. If $k=1$, this follows from 2.1(i), for $p_{*}<q_{k}=q_{1}$ while $q_{0 *} \nless q_{1}$. If $k>1$, then $q_{k} \$ \kappa\left(q_{0}\right)$ by 2.1(iv), whence $q_{0 *}=p_{*}$ would imply $q_{0} \leqslant q_{0 *}+q_{k *}=$ $q_{k *}$. But then we would have $q_{0} \leqslant q_{k} \kappa(p)=p_{*}=q_{0 *}$, a contradiction.

Thus we may choose $j \geqslant 1$ minimal with respect to the property $q_{j *} \geqslant p_{*}$. We want next to apply a variation of Lemma 2.4 to show that $\Sigma_{i<j} q_{i} \leqslant \kappa(p)$

So assume $\Sigma_{i<j} q_{i} \$ \kappa(p)$, in which case of course $j>1$. We may then follow almost exactly the proof of Lemma 2.4, except that in applying Lemma 2.3, choose $b=\sum_{i<j} q_{i *}$ and $b_{0}=\sum_{i<j-1} q_{i *}+q_{j-1}$. All subsequent references to $p_{* *}$ in the proof must then be omitted or have $p_{* *}$ replaced by $p_{*}$, as appropriate. The lone exception to this rule is that in order to show $b \neq q_{0 *}$ in the proof of (i)', observe that $b=\sum_{i<j} q_{i *} \$ \kappa(p)$ by the free star principle, while $q_{0 *} \leqslant \kappa(p)$ by assumption. The details of verifying that this all works will be left to the reader.

We conclude from this argument that $\sum_{i<j} q_{i} \leqslant \kappa(p)$. If $j=k$, this is the desired result. So assume $j<k$ and $\Sigma_{i<k} q_{i} \$ \kappa(p)$, and let us apply Lemma 2.7 with $t=\kappa(p)^{*}\left[p+\sum_{i<j} q_{i *}\right]$. Hypotheses (i)-(iii) of Lemma 2.7 hold immediately, while (vii) is a consequence of $q_{0} \leqslant \kappa(p)$. This leaves hypotheses (iv)-(vi) for us to verify.

(iv) Clearly $p \leqslant t \leqslant \kappa(p)^{*}$. Note $q_{j-1 *} \leqslant t \kappa(p)$. Therefore we cannot have $p=t$, for that would imply $q_{j-1 *} \leqslant p \kappa(p)=p_{*} \leqslant q_{j}$, contrary to 2.1(i). Hence $p<t$.

(v) Since $p_{*} \leqslant q_{j *} \leqslant \kappa\left(q_{j-1}\right)$, the free star principle yields $p \leqslant \kappa\left(q_{j-1}\right)$. Combining this with 2.1(ii) and (iii), we have $t \leqslant p+\Sigma_{i<j} q_{i *} \leqslant \kappa\left(q_{j-1}\right)$, as desired.

(vi) If $t \leqslant p+\Sigma_{i<j-1} q_{i *}+q_{j-1}$, then we may apply (W) to the inclusion

$$
\kappa(p)^{*}\left[p+\sum_{i<j} q_{i *}\right] \leqslant p+\sum_{i<j-1} q_{i *}+q_{j-1} .
$$

If $\kappa(p)^{*}$ is below the right-hand side, then $q_{k} \leqslant \kappa(p)^{*} \leqslant p+\sum_{i<j-1} q_{i} \leqslant \kappa\left(q_{k}\right)$ using 2.1(ii), a contradiction. Skipping over the second possible inclusion for a moment, note that the left-hand side $t \$ p$ since $p<t$ was shown above in (iv). Likewise, $p_{*}<t$ implies $t \$ q_{i *}$ for $i \leqslant j-1$ by the choice of $j$, while $t \neq q_{j-1}$ as $t \leqslant \kappa\left(q_{j-1}\right)$ by (v) above.

So suppose that the second term, and hence in particular $q_{j *}$, is below the right-hand side. Recall that $j<k$, whence 2.1 (iii) implies $q_{j *}=q_{j}\left(q_{j *}+q_{j+1 *}\right)$. Then we may apply $(W)$ to the inclusion

$$
q_{j}\left(q_{j *}+q_{j+1 *}\right) \leqslant p+\sum_{i<j-1} q_{i *}+q_{j-1} .
$$

Since $p_{*} \leqslant q_{j *} \leqslant \kappa\left(q_{j}\right)$, by the free star principle $p \leqslant \kappa\left(q_{j}\right)$. As also $\Sigma_{i<j-1} q_{i *}+$ $q_{j-1} \leqslant \kappa\left(q_{j}\right)$ by 2.1 (ii), we have $q_{j} \$ p+\sum_{i<j-1} q_{i *}+q_{j-1}$.

Suppose $q_{j+1 *} \leqslant p+\sum_{i<j-1} q_{i *}+q_{j-1}$. Then, using 2.1(iv), we obtain

$$
u \equiv p+\sum_{i<j-1} q_{i *}+q_{j-1}=p+\sum_{i<j-1} q_{i *}+q_{j+1 *}
$$


whence, by $\left(\mathrm{SD}_{\bigvee}\right), u=p+\sum_{i \leqslant j-1} q_{i *}+q_{j-1} q_{j+1 *}$. Since $q_{j-1}<u$, we have $u \nless \kappa\left(q_{j-1}\right)$. However, $p+\sum_{i \leqslant j-1} q_{i *} \leqslant \kappa\left(q_{j-1}\right)$ as in (v) above, so that $q_{j-1} q_{j+1 *}$ $\$ \kappa\left(q_{j-1}\right)$, which can only happen if $q_{j-1} \leqslant q_{j+1 *}$. It follows from this that $j+1<$ $k$, for otherwise (i.e., if $j+1=k$ ) we would have $q_{j-1 *}<q_{j-1}<q_{k *} \kappa(p)=p_{*}<$ $q_{j}$, contrary to 2.1(i). But then $q_{j+1 *}=q_{j+1}\left(q_{j+1 *}+q_{j+2 *}\right)$, and

$$
q_{j+1}\left(q_{j+1 *}+q_{j+2 *}\right) \leqslant p+\sum_{i<j-1} q_{i *}+q_{j-1}
$$

is a W-failure. To see this, first note that $p_{*} \leqslant q_{j *} \leqslant \kappa\left(q_{j+1}\right)$, whence by the free star principle $p \leqslant \kappa\left(q_{j+1}\right)$. Thus the whole right-hand side is below $\kappa\left(q_{j+1}\right)$, and not above $q_{j+1}$. Likewise the right-hand side is below $\kappa\left(q_{j}\right)$ while, by 2.1 (iv), $q_{j+2 *}$ $\kappa\left(q_{j}\right)$. Therefore $q_{j+2 *} \$ p+\Sigma_{i<j-1} q_{i *}+q_{j-1}$. Now considering the part of the right-hand side obtained by replacing $q_{j-1}$ with $q_{j-1 *}$, note $p+\Sigma_{i<j-1} q_{i *}+q_{j-1 *}$ $\leqslant \kappa\left(q_{j-1}\right)$, while by 2.1 (iv) the left-hand side $q_{j+1 *} \$ \kappa\left(q_{j-1}\right)$. Thus $q_{j+1 *}$ is not below any of those terms, which leaves us to deal with the only remaining possibility, $q_{j+1 *}=q_{j-1}$. Supposing that to hold, we have $q_{j+1 *}<\kappa(p)$, whence by the free star principle $q_{j+1} \leqslant \kappa(p)$. Note also that $q_{j+1 *} \neq p_{*}$, for $p_{*} \nless q_{j+1 * *}=$ $q_{j-1 *}$ by the choice of $j$, and $p_{*} \neq q_{j+1 *}=q_{j-1}$ since $p_{*} \leqslant q_{j *}$ and $q_{j-1} \nless q_{j *}$ (as a consequence of 2.1(i)). Thus, using induction, we may apply our lemma to the shorter $B$-type sequence $\left\langle q_{j+1}, \ldots, q_{k}\right\rangle$ to obtain $\Sigma_{j+1<i<k} q_{i} \leqslant \kappa(p)$. Now also we are assuming $\Sigma_{i<j} q_{i} \leqslant \kappa(p)$ and $\Sigma_{i<k} q_{i} \$ \kappa(p)$. Therefore $q_{j} \$ \kappa(p)$, and since $p_{*} \leqslant q_{j}$ that means $p \leqslant q_{j}$. In fact, $p<q_{j}$ since elements of a minimal cycle are of course distinct. Note that $j+1<k$ since $q_{j+1} \leqslant \kappa(p)$ and $q_{k} \$ \kappa(p)$. Hence, using 2.1(iv) and ( $\dagger$ ), we may calculate

$$
q_{j} \leqslant q_{j *}+q_{k *} \leqslant\left[p+\sum_{i<j-1} q_{i *}+q_{j-1}\right]+\kappa(p)^{*}=\kappa(p)^{*} .
$$

Thus we have shown that $q_{j} A p$, contrary to the minimality of our cycle. We conclude that $q_{j+1 *} \$ p+\sum_{i<j-1} q_{i *}+q_{j-1}$.

We are not finished yet with the inclusion ( $\dagger$ ), as we must show that $q_{j *}$ is not below one of the terms on the right-hand side. Now $q_{j *} \nless q_{i *}$ for $i<j-1$ since $j$ is minimal such that $p_{*} \leqslant q_{j *}$, and of course $q_{j *} \neq q_{j-1}$ by 2.1(i). Suppose $q_{j *} \leqslant p$. Then since $p_{*} \leqslant q_{j *}$ we have either $q_{j *}=p$ or $q_{j *}=p_{*}$. Also this implies $j<k-$ 1, for by 2.1(i) we cannot have $q_{k-1 *} \leqslant p<q_{k}$. Hence, using 2.1(iv), $q_{j}<q_{j *}+q_{k *}$ $\leqslant p+q_{k *}=q_{k *}$. In case $q_{j *}=p$, this means $p<q_{j}<q_{k} \leqslant \kappa(p)^{*}$, so that $q_{j} A p$, contrary to the minimality of our cycle. But if $q_{j *}=p_{*}$, then $q_{j}<\kappa(p)$ since $p_{*} \leqslant q_{j}$ and $p \$ q_{j}$, so we have $q_{j} \leqslant q_{k} \kappa(p)=p_{*}=q_{j *}$, a contradiction. Therefore $q_{j *} \$ p$.

We conclude that $(\dagger)$ does not hold. Hence hypothesis (vi) of Lemma 2.7 is valid, and that lemma applies to yield $\Sigma_{i<j} q_{i *} \$ \kappa(p)$. This, however, contradicts the conclusion which we obtained earlier by applying a modification of Lemma 2.4. Therefore $\Sigma_{i<k} q_{i} \leqslant \kappa(p)$, as desired.

Lemma 3.6. Let $\left\langle q_{0}, \ldots, q_{k}\right\rangle$ with $k \geqslant 1$ be a B-type sequence, and let $q_{k} A_{2} p$. If $q_{0} \leqslant \kappa(p)$ and $q_{0 *} \ngtr p_{*}$, then $p=q_{k *}$. 
Proof. Suppose $p<q_{k *}$, and let us apply Lemma 2.7 with $t=q_{k *}$ and $j=k$ (so there is no minimality condition assumed on $j$ this time). As in the proof of Lemma 3.5 , we may assume $q_{0 *} \ngtr p_{*}$, which is hypothesis 2.7(ii). Of the remaining hypotheses, (i), (iii)-(v) and (vii) follow immediately from our assumptions, leaving (vi) for us to verify.

If (vi) fails, we have

$$
q_{k *}=q_{k} \kappa\left(q_{k}\right) \leqslant p+\sum_{i<k-1} q_{i *}+q_{k-1},
$$

in which case we may apply (W). Now the right-hand side is below $\kappa\left(q_{k}\right)$ by 2.1(ii); hence $q_{k} \$ p+\sum_{i<k-1} q_{i *}+q_{k-1}$. Skipping over the second possible inclusion for a moment, we note that $q_{k *} \$ p$ since $p<q_{k *}$ by assumption. For $i<k-1$, 2.1(iv) says that $q_{k *} \$ \kappa\left(q_{i}\right)$, whence $q_{k *} \$ q_{i *}$. Moreover $q_{k *} \$ q_{k-1}$, for 2.1(i) implies $q_{k *} \neq q_{k-1}$, whence we need only show $q_{k *} \$ q_{k-1 *}$. If $k=1, q_{1 *} \$ q_{0 *}$ follows from $p_{*}<q_{1 *}$ and $p_{*} \$ q_{0 *}$; while if $k>1, q_{k *} \$ q_{k-1 *}$ because $q_{k *}$ $\kappa\left(q_{k-2}\right)$ by 2.1 (iv) and $q_{k-1 *} \leqslant \kappa\left(q_{k-2}\right)$ by 2.1 (iii).

So suppose $\kappa\left(q_{k}\right) \leqslant p+\sum_{i<k-1} q_{i *}+q_{k-1}$, whence by 2.1(ii), $\kappa\left(q_{k}\right)=p+$ $\Sigma_{i<k-1} q_{i *}+q_{k-1}$. Then we claim that $\kappa(p)\left[\sum_{i<k} q_{i}\right] \nless \kappa\left(q_{k}\right)$, for otherwise we could apply (W) to the inclusion $\kappa(p)\left[\sum_{i<k} q_{i}\right] \leqslant p+\sum_{i<k-1} q_{i *}+q_{k-1}$ to obtain a contradiction. For $\kappa(p)$ is not below $\kappa\left(q_{k}\right)$, since $q_{k} A_{2} p$ implies $\kappa\left(q_{k}\right) B^{d} \kappa(p)$, which makes these elements incomparable. Of course $q_{k} \nless \kappa\left(q_{k}\right)$, wherefore $\Sigma_{i<k} q_{i}$ $\$ \kappa\left(q_{k}\right)$. Observe, using Lemma 3.5, that $p_{*}+\sum_{i<k} q_{i} \leqslant \kappa(p)\left[\sum_{i<k} q_{i}\right]$, whence it will suffice to show that $p_{*}+\sum_{i<k} q_{i}$ is not below any term on the right-hand side. Since $p<q_{k}$ and $q_{k-1} \$ q_{k}$ (by 2.1(i)), $p_{*}+\Sigma_{i<k} q_{i} \$ p$. Because $p_{*} \$ q_{0 *}$, and $q_{i-1} \$ q_{i *}$ for $i>0$ (by 2.1(i) again), we have $p_{*}+\Sigma_{j<k} q_{k} \$ q_{i *}$ for $0 \leqslant i<k-$ 1 . It remains to show that $p_{*}+\Sigma_{i<k} q_{i} \$ q_{k-1}$. If $k=1$, note $p_{*} \neq q_{0}$ since $p_{*}<q_{k}=q_{1}$ and $q_{0} \$ q_{1}$; combined with $p_{*} \$ q_{0 *}$ this yields $p_{*} \$ q_{0}=q_{k-1}$. If $k>1$, the statement follows from $q_{k-2} \$ q_{k-1}$.

Therefore, using the above claim, Lemma 1.1(ii) and Lemma 3.5, we calculate that

$$
\sum_{i<k} q_{i}=q_{k *}+\kappa(p)\left[\sum_{i<k} q_{i}\right] .
$$

Hence by $\left(\mathrm{SD}_{\bigvee}\right)$, since $q_{k} \kappa(p)=p_{*}<q_{k *}, \quad \sum_{i<k} q_{i}=\sum_{i<k} q_{i}+q_{k *} \leqslant \kappa\left(q_{k}\right)$, which is of course a contradiction.

Thus $\kappa\left(q_{k}\right) \$ p+\sum_{i<k-1} q_{i *}+q_{k-1}$, so hypothesis (vi) of Lemma 2.7 holds. We conclude by that lemma that $\Sigma_{i<k} q_{i *} \$ \kappa(p)$, which contradicts Lemma 3.5. Hence $p=q_{k *}$.

Lemma 3.7. Let $\left\langle q_{0}, \ldots, q_{k}\right\rangle$ with $k \geqslant 1$ be a $B$-type sequence, and let $q_{k} A_{2} p_{0} B p_{1}$. If $q_{0} \leqslant \kappa\left(p_{0}\right)$ and $q_{0 *} \ngtr p_{0 *}$, then $\Sigma_{i<k} q_{i} \leqslant p_{0}+p_{1}$.

Proof. Supposing that $\Sigma_{i<k} q_{i} \$ p_{0}+p_{1}$, we will apply Lemma 2.3. Let

$$
\begin{aligned}
& a=q_{k}, \quad a_{0}=\left(\sum_{i<k} q_{i}\right)\left(p_{0}+p_{1}\right), \\
& b=p_{0 *}+p_{1 *}, \quad b_{0}=\kappa\left(p_{0}\right)\left[p_{0}+p_{1}\right] .
\end{aligned}
$$


Choose $a_{1}$ such that $a \leqslant a_{1} \prec a_{0}$. (We will show in (ii) below that this is possible. The argument for (v) below shows that $a_{0}$ is meet-reducible, whence $a_{0} \in J(L)$ and $a_{1}=a_{0 *}$.)

Now let us check conditions (i)' and (ii)-(vii) of Lemma 2.3.

(i) Certainly $a=q_{k} \in J(L)$. Note that by Lemma 3.6, $a_{*}=q_{k *}=p_{0}$. We will argue below that $p_{1 *} \$ p_{0 *}$ (i.e., $p_{0} B_{1} p_{1}$ ). On the one hand, this will show that $b$ is join-reducible, since $p_{0 *} \$ p_{1 *}$ by 2.1 (i). On the other hand, it also enables us to apply Lemma 7.3 of [15] to obtain $q_{k} \leqslant p_{0}+p_{1}$. (To prove this claim directly, apply Lemma 2.5 with $a=p_{0}+p_{1}\left(=p_{0 *}+p_{1}\right), b=\kappa\left(p_{0}\right), c=q_{k}$.) Since $p_{0}=$ $q_{k *} \leqslant \kappa\left(q_{k}\right)$, this means that $p_{1} \$ \kappa\left(q_{k}\right)$, whence by the free star principle $p_{1 *} \leqslant$ $\kappa\left(q_{k}\right)$. Therefore $q_{k} \leqslant q_{k *}+p_{1 *}=p_{0}+p_{1 *}$, i.e., $a \leqslant a_{*}+b$.

Assume $p_{1 *} \leqslant p_{0 *}$. Let us apply (W) to the inclusion

$$
q_{k}\left(p_{0}+q_{k-1}\right)=p_{0} \leqslant p_{0 *}+p_{1} .
$$

(This is easily verified using 2.1(ii), Lemma 3.6 and $p_{0} B p_{1}$.) If $q_{k}<p_{0 *}+p_{1}$, then as above $q_{k} \leqslant p_{0}+p_{1 *}=p_{0}<q_{k}$, a contradiction. If $q_{k-1} \leqslant p_{0 *}+p_{1}$, then since $p_{0 *}<q_{k *} \leqslant \kappa\left(q_{k-1}\right)$ by 2.1 (iii), we must have $p_{1} \leqslant \kappa\left(q_{k-1}\right)$. But then the free star principle yields $p_{1 *} \leqslant \kappa\left(q_{k-1}\right)$, contrary to $p_{1 *} \leqslant p_{0 *} \leqslant \kappa\left(q_{k-1}\right)$. Of course $p_{0} \leqslant p_{0 *}$, and $p_{0} \$ p_{1}$ because $p_{0} B p_{1}$ (see Figure 1). Thus the assumption $p_{1 *} \leqslant p_{0 *}$ leads to a W-failure, wherefore we conclude $p_{1 *} \$ p_{0 *}$.

(ii) By what we have done so far, $a \leqslant a_{0}$. To see that $a \neq a_{0}$, suppose otherwise, in which case we may apply (W) to the inclusion

$$
\left(\sum_{i<k} q_{i}\right)\left(p_{0}+p_{1}\right)=q_{k} \leqslant p_{0}+p_{1 *} .
$$

We are assuming that $\sum_{i \leqslant k} q_{i} \$ p_{0}+p_{1}$, and $p_{1} \$ p_{0}+p_{1 *}$ since $p_{0}+p_{1 *} \leqslant \kappa\left(p_{1}\right)$ by 2.1 (ii) (or see Figure 1). On the other hand, $q_{k} \$ p_{0}=q_{k *}$ and $q_{k} \$ p_{1 *}$, for else $p_{0 *}<q_{k}<p_{1}$, contrary to 2.1(i). Thus the assumption $a=a_{0}$ leads to a W-failure. We conclude that $a<a_{0}$, and $a_{1}$ may be chosen with $a \leqslant a_{1}<a_{0}$.

(iii) Clearly $b \leqslant b_{0}$, for by definition $p_{0} B p_{1}$ implies $p_{1 *} \leqslant \kappa\left(p_{0}\right)$.

(iv) If $a \leqslant b_{0}$, we would have $p_{0}<q_{k}=a \leqslant b_{0} \leqslant \kappa\left(p_{0}\right)$, a contradiction. Thus $a \$ b_{0}$.

(v) If $b \leqslant a_{0}$, then $p_{1 *} \leqslant \sum_{i \leqslant k} q_{i}$. Therefore, using $q_{k} \leqslant p_{0}+p_{1 *}$, we see that $\sum_{i<k} q_{i}=\sum_{i<k} q_{i}+p_{0}+p_{1 *}$, whence by $\left(\mathrm{SD}_{\vee}\right)$

$$
\sum_{i<k} q_{i}=\sum_{i<k} q_{i}+p_{0}+q_{k} p_{1 *}
$$

However, $q_{k} \$ p_{1 *}$ since $p_{0}<q_{k}$ and $p_{0} \leqslant p_{1 *}$, whence $q_{k} p_{1 *} \leqslant q_{k *}=p_{0}$. Thus, using 2.1(ii), $\sum_{i \leqslant k} q_{i}=\sum_{i<k} q_{i}+p_{0} \leqslant \kappa\left(q_{k}\right)$, a contradiction. Hence $b \$ a_{0}$.

(vi) If $a_{0} \leqslant a_{1}+b_{0}$, then we may apply (W) to the inclusion

$$
\left(\sum_{i<k} q_{i}\right)\left(p_{0}+p_{1}\right) \leqslant a_{1}+\kappa\left(p_{0}\right)\left[p_{0}+p_{1}\right]
$$


Now $\Sigma_{i<k} q_{i}$ is not below the right-hand side, since $a_{1}+\kappa\left(p_{0}\right)\left[p_{0}+p_{1}\right]<p_{0}+p_{1}$, while by assumption $\Sigma_{i \leqslant k} q_{i} \$ p_{0}+p_{1}$. If $p_{1} \leqslant a_{1}+b_{0} \leqslant a_{0}+b_{0}$, then we may easily see that $p_{0}+p_{1}=a_{0}+b_{0}$, i.e.,

$$
p_{0}+p_{1}=\left(\sum_{i<k} q_{i}\right)\left(p_{0}+p_{1}\right)+\kappa\left(p_{0}\right)\left[p_{0}+p_{1}\right] .
$$

Applying $\left(\mathrm{SD}_{\vee}\right)$ to this, we obtain

$$
p_{0}+p_{1}=p_{0}+p_{1}\left(\sum_{i<k} q_{i}\right)+p_{1} \kappa\left(p_{0}\right) .
$$

But $p_{1} \$ \sum_{i \leqslant k} q_{i}$ by the argument of (v), and $p_{1} \$ \kappa\left(p_{0}\right)$ by the definition of $p_{0} B p_{1}$ (although $\left.p_{1 *} \leqslant \kappa\left(p_{0}\right)\right)$. Therefore $p_{1}\left(\Sigma_{i \leqslant k} q_{i}\right)+p_{1} \kappa\left(p_{0}\right)=p_{1 *}$, and $p_{0}+p_{1}=p_{0}+$ $p_{1 *} \leqslant \kappa\left(p_{1}\right)$, a contradiction. Hence $p_{1} \$ a_{1}+b_{0}$. Continuing with (W), of course the left-hand side $a_{0} \$ a_{1}$, and since $a \leqslant a_{0}$, we have $a_{0} \nless b_{0}$ by (iv). We conclude that $a_{0} \$ a_{1}+b_{0}$.

(vii) If $b_{0} \leqslant a_{1}+b$, then we may apply (W) to the inclusion

$$
\kappa\left(p_{0}\right)\left[p_{0}+p_{1}\right] \leqslant a_{1}+p_{1 *}
$$

since $p_{0 *}<a \leqslant a_{1}$. If $\kappa\left(p_{0}\right) \leqslant a_{1}+p_{1 *}$, then using Lemma 3.5 we have $\Sigma_{i<k} q_{i} \leqslant$ $\kappa\left(p_{0}\right) \leqslant a_{1}+p_{1 *} \leqslant p_{0}+p_{1}$. Since also $q_{k} \leqslant p_{0}+p_{1}$, this implies $\Sigma_{i<k} q_{i} \leqslant p_{0}+$ $p_{1}$, contrary to assumption. If $p_{1} \leqslant a_{1}+p_{1 *}=a_{1}+b$, then again we would obtain $p_{1}+p_{0}=a_{0}+b_{0}$, a possibility eliminated in the argument of (vi). On the other hand, since $b \leqslant b_{0}$ and $a_{1}<a_{0}$, whereas $b \leqslant a_{0}$ by (v), we have $b_{0} \$ a_{1}$. Finally, $p_{0 *} \leqslant \kappa\left(p_{0}\right)\left[p_{0}+p_{1}\right]$ while $p_{0 *} \$ p_{1}$, whence $b_{0} \$ p_{1 *}$.

We conclude from Lemma 2.3 that this situation cannot occur in an $S$-lattice, whence $\Sigma_{i \leqslant k} q_{i} \leqslant p_{0}+p_{1}$.

With these preliminaries out of the way, we are now in a position to take care of the case when $q_{0 *} \ngtr p_{* *}$. Roughly speaking, what we will show is this. If $\left\langle q_{0}, \ldots, q_{k}\right\rangle$ is a $B$-type sequence which is either a sequence of $B$ 's or arises from previous applications of this lemma, and if $q_{k} A_{2} p_{0} B p_{1} B \ldots B p_{m}$, and if furthermore $q_{0 *} \ngtr p_{0 * *}$ (so that in particular, Lemma 3.4 does not apply), then by dropping $q_{k}$ we can obtain a new, longer $B$-type sequence $\left\langle q_{0}, \ldots, q_{k-1}, p_{0}, \ldots, p_{m}\right\rangle$. (Of course, we still do not have a minimal cycle without $q_{k}$, for $q_{k-1} C p_{0}$ does not hold.) More precisely:

LEMMA 3.8. Let

$$
\begin{array}{cccccc} 
& r_{10} & B & \ldots & B & r_{1 k_{1}} \\
A_{2} & r_{20} & B & \ldots & B & r_{2 k_{2}} \\
& & & \vdots & & \\
& & & \vdots & & \\
A_{2} & r_{n 0} & B & \ldots & B & r_{n k_{n}},
\end{array}
$$


with $n \geqslant 1$ and $k_{i} \geqslant 1$ for $1 \leqslant i \leqslant n$, be a subsequence of a minimal cycle in an $S$-lattice (where the above notation means that $r_{i k_{i}} A_{2} r_{i+1,0}$ for $1 \leqslant i<n$ ). If $r_{10} \leqslant$ $\kappa\left(r_{i 0}\right)$ and $r_{10 *} \ngtr r_{i 0 *}$ for $1<i \leqslant n$, then the sequence obtained by omitting every $r_{i k_{i}}$ with $1 \leqslant i<n$,

$$
\left\langle r_{10}, \ldots, r_{1, k_{1}-1}, r_{20}, \ldots, r_{i, k_{i}-1}, r_{i+1,0}, \ldots, r_{n k_{n}}\right\rangle
$$

is a B-type sequence.

Proof. We proceed by induction on $n$. If $n=1$, then there are no $A_{2}$ 's, and our lemma reduces to Lemma 2.1. Thus we may assume that $n>1$ and the initial segment $\left\langle r_{10}, \ldots, r_{i, k_{i}-1}, r_{i+1,0}, \ldots, r_{n-1, k_{n-1}}\right\rangle$ of (††) is a $B$-type sequence. (This is the inductive hypothesis to which we will refer thoughout the proof.) We must verify that conditions (i)-(iv) of Lemma 2.1 hold for our longer sequence.

Condition (i) requires that we show $p_{*} \$ q$ for each consecutive pair $p, q$ from our sequence. This follows from the inductive hypotheses for consecutive pairs with $p$ and $q$ between $r_{10}$ and $r_{n-1, k_{n-1}-1}$, and from Lemma 2.1(i) for pairs between $r_{n 0}$ and $r_{n k_{n}}$. For the remaining pair, we have $r_{n-1, k_{n-1}-1 *} \$ r_{n 0}$, because $r_{n-1, k_{n-1}-1} B r_{n-1, k_{n-1}} A_{2} r_{n 0}$ implies $r_{n 0}<r_{n-1, k_{n-1}}$ but $r_{n-1, k_{n-1}-1 *} \$ r_{n-1, k_{n-1}}$.

For (ii), we must show that for any $q$ in our sequence

$$
\sum\{p: p \text { precedes } q\} \leqslant \kappa(q) \text {. }
$$

Again, for $q$ between $r_{10}$ and $r_{n-1, k_{n-1}-1}$ this follows from the inductive hypothesis. For $q=r_{n 0}$, Lemma 3.5 applies to yield the desired result. But for $q=r_{n i}$ $\left(1 \leqslant i \leqslant k_{n}\right)$, note that Lemma 1.3 (ii) implies $\kappa\left(r_{n, i-1}\right) \leqslant \kappa\left(r_{n i}\right)$, whence we may calculate (using induction and 2.1(ii))

$$
\begin{aligned}
\sum\left\{p: p \text { precedes } r_{n i}\right\} & =\sum\left\{p: p \text { precedes } r_{n, i-1}\right\}+r_{n, i-1} \\
& \leqslant \kappa\left(r_{n, i-1}\right)+r_{n, i-1} \leqslant \kappa\left(r_{n i}\right) .
\end{aligned}
$$

Condition (iii) requires that for each consecutive pair $p, q$ from our sequence $q_{*} \leqslant \kappa(p)$. As in (i), every pair except $r_{n-1, k_{n-1}-1}, r_{n 0}$ is covered by either the inductive hypothesis or Lemma 2.1(iii). For this pair, we simply note that $r_{n 0 *}<$ $r_{n-1, k_{n-1} *} \leqslant \kappa\left(r_{n-1, k_{n-1}-1}\right)$, as desired.

For (iv), we must show that $q_{*} \$ \kappa(p)$ whenever $p$ precedes $q$ by at least two places in our sequence. If both $p$ and $q$ lie between $r_{10}$ and $r_{n-1, k_{n-1}-1}$, or between $r_{n 0}$ and $r_{n k_{n}}$, then this is immediate. Therefore we may assume that $p=r_{i m}$ for some $i<n$, and $q=r_{n j}$.

If $j=0$, let $r_{i m}$ precede $r_{n-1, k_{n-1}-1}$, and note that Lemma 3.6 applies to yield $r_{n 0}=r_{n-1, k_{n-1} *}$. Since the segment $\left\langle r_{10}, \ldots, r_{s, n_{3}-1}, r_{s+1,0}, \ldots, r_{n-1, k_{n-1}}\right\rangle$ is a $B$ type sequence, we have $r_{n 0}=r_{n-1, k_{n-1} *} \$ \kappa\left(r_{i m}\right)$, whence by the free star principle $r_{n 0 *} \$ \kappa\left(r_{\text {im }}\right)$.

So assume $j \geqslant 1$. Since $\kappa\left(r_{i 0}\right) \leqslant \kappa\left(r_{i 1}\right) \leqslant \cdots \leqslant \kappa\left(r_{i, k_{i}-1}\right)$ by Lemma 1.3(ii), while $r_{i k_{i}}$ is not in our sequence, it suffices to show that $r_{n j *} \$ \kappa\left(r_{i, k_{i}-1}\right)$ for each $i<n$. Moreover, by virtue of the free star principle, we need only show that $r_{n j} \nless \kappa\left(r_{i, k_{i}-1}\right)$ for $i<n$. 
If $j=1$ and $i=n-1$, we may apply Lemma 3.7 to obtain $r_{n-1, k_{n-1}-1} \leqslant r_{n 0}+$ $r_{n 1}$. Since $r_{n 0}=r_{n-1, k_{n-1} *} \leqslant \kappa\left(r_{n-1, k_{n-1}-1}\right)$, we must have $r_{n 1} \leqslant \kappa\left(r_{n-1, k_{n-1}-1}\right)$. Thus we may assume that either $j>1$ or $i<n-1$.

The final argument for (iv) is based on the following special case. If $r_{i+1,0} \leqslant r_{n j *}$, then $r_{n j} \$ \kappa\left(r_{i, k_{i}-1}\right)$. To prove this claim, suppose that $r_{i+1,0} \leqslant r_{n j *}$ and $r_{n j} \leqslant$ $\kappa\left(r_{i, k_{i}-1}\right)$, and let us apply Lemma 2.7 with the following substitutions.

$$
\begin{aligned}
\left\langle q_{1}, \ldots, q_{k}\right\rangle & \leftrightarrow\left\langle r_{10}, \ldots, r_{s, n_{3}-1}, r_{s+1,0}, \ldots, r_{i k_{i}}\right\rangle, \quad p \leftrightarrow r_{i+1,0}, \\
t & \leftrightarrow \kappa\left(r_{i+1,0}\right)^{*}\left[r_{i, k_{i}-1 *}+r_{n j}\right], \quad j \leftrightarrow\left(i, k_{i}\right) .
\end{aligned}
$$

Condition 2.7(i) follows from our inductive hypothesis. For 2.7(ii), note that $r_{i+1,0 *} \nless r_{10 *}$ by one of the hypotheses of Lemma 3.8, while equality is excluded by the remarks beginning the proof of Lemma 3.5. Condition 2.7(iii) is a consequence of $r_{i k_{i}} A r_{i+1,0}$.

For 2.7 (iv), we clearly have $r_{i+1,0} \leqslant \kappa\left(r_{i+1,0}\right)^{*}\left[r_{i, k_{i}-1 *}+r_{n j}\right] \leqslant \kappa\left(r_{i+1,0}\right)^{*}$ since $r_{i+1,0}<r_{n j}$. Moreover, the first inequality is strict because $r_{i, k_{i}-1 *} \leqslant$ $\kappa\left(r_{i+1,0}\right) *\left[r_{i, k_{i}-1 *}+r_{n j}\right]$ by Lemma 3.5, while $r_{i, k_{i}-1 *} \$ r_{i+1,0}=r_{i, k_{i} *}$ by 2.1(i).

Condition 2.7(v) follows from our assumption that $r_{n j} \leqslant \kappa\left(r_{i, k_{i}-1}\right)$.

If 2.7(vi) fails, then we may apply (W) to the inclusion

$$
\kappa\left(r_{i+1,0}\right)^{*}\left[r_{i, k_{i}-1 *}+r_{n j}\right] \leqslant r_{i+1,0}+\sum\left\{r_{\alpha \beta *}: r_{\alpha \beta} \text { precedes } r_{i, k_{i}-1}\right\}+r_{i, k_{i}-1} \text {. }
$$

Now $\kappa\left(r_{i+1,0}\right)^{*}$ is not below the right-hand side (RHS), since $r_{i, k_{i}} \leqslant \kappa\left(r_{i+1,0}\right)^{*}$ while by the inductive hypothesis (using $2.1\left(\right.$ ii)) RHS $\leqslant \kappa\left(r_{i, k_{i}}\right)$. Similarly, since RHS $\leqslant$ $\kappa\left(r_{n j}\right)$ by condition 2.1(ii), which was proved above, we have $\left[r_{i, k_{i}-1 *}+r_{n j}\right] \nless$ RHS. On the other hand, recall from 2.7 (iv) that $r_{i+1,0}$ is strictly below the left-hand side (LHS). Thus LHS $\$ r_{i+1,0}$. Moreover, since $r_{i+1,0}=r_{i, k_{i} *} \$ \kappa\left(r_{\alpha \beta}\right)$ for $r_{\alpha \beta}$ preceding $r_{i, k_{i}-1}$ by 2.1 (iv) of the inductive hypothesis, we have LHS $\$ r_{\alpha \beta *}$. Finally, suppose LHS $\leqslant r_{i, k_{i}-1}$, whence $r_{i+1,0}=r_{i k_{i} *} \leqslant r_{i, k_{i}-1}$. Then $r_{i, k_{i}-1} B_{2} r_{i k_{i}}$. It is easy to see that in a sequence of $B$ 's, only the first $B$ can be a $B_{2}$. (For if $p B q B u$ and $u_{*} \leqslant q$, then $u_{*} \neq q$, so $u_{*} \leqslant q_{*} \leqslant \kappa(p)$, contrary to 2.1(iv). This is Lemma 7.6 of [15].) Therefore $k_{i}=1$. Then $i \neq 1$, for if $r_{10} B_{2} r_{11} A_{2} r_{20}$ we would have $r_{20 *}<r_{11 *}<$ $r_{10 *}$, contrary to one of our original hypotheses. However, if $i>1$ we have $r_{i 0} B_{1} r_{i 1}$, because by the inductive hypothesis $r_{i 0 *}<\kappa\left(r_{i-1, k_{i-1}-1}\right)$ and $r_{i 1 *} \nless$ $\kappa\left(r_{i-1, k_{i-1}-1}\right)$, whence $r_{i 1 *} \$ r_{i 0 *}$. Thus LHS $\$ r_{i, k_{i-1}}$, and 2.7(vi) holds.

For 2.7(vii), $r_{10} \leqslant \kappa\left(r_{i+1,0}\right)$ implies $r_{i+1,0} B r_{10}$ does not hold.

Thus Lemma 2.7 applies to yield $\Sigma\left\{r_{\alpha \beta *}: r_{\alpha \beta}\right.$ precedes $\left.r_{i k_{i}}\right\} \$ \kappa\left(r_{i+1,0}\right)$. This, however, contradicts property 2.1 (ii), which we have already proved. Therefore $r_{n j} \$ \kappa\left(r_{i, k_{i}-1}\right)$ whenever $r_{i+1,0} \leqslant r_{n j *}$, as claimed.

Now fix $j \geqslant 1$. If 2.1(iv) fails with $q=r_{n j}$, then we may choose $i<n$ maximal such that $r_{n j} \leqslant \kappa\left(r_{i, k_{i}-1}\right)$. Since $\kappa\left(r_{i, k_{i}-1}\right) \leqslant \kappa\left(r_{i k_{i}}\right)$ by Lemma 1.3(ii), we have $r_{n j} \leqslant$ $\kappa\left(r_{i k_{i}}\right)$, so that $r_{i k_{i}} \$ r_{i k_{i} *}+r_{n j}=r_{i+1,0}+r_{n j}$. Thus $r_{i k_{i}}\left(r_{i+1,0}+r_{n j}\right)=r_{i+1,0}$. On the other hand, $r_{n j *} \$ \kappa\left(r_{i+1,0}\right)$. If $i<n-1$, this follows from the maximality of $i$, the fact that $\kappa\left(r_{i+1,0}\right) \leqslant \kappa\left(r_{i+1, k_{i+1}-1}\right)$, and the free star principle. If $i=n-1$, we have shown that $j>1$, whence $r_{n j *} \$ \kappa\left(r_{n 0}\right)$ follows from 2.1(iv). Thus $r_{i+1,0} \leqslant r_{i+1,0 *}+$ $r_{n j *^{*}}$. Combining these relations, we obtain the inclusion

$$
r_{i k_{i}}\left(r_{i+1,0}+r_{n j}\right)=r_{i+1,0} \leqslant r_{i+1,0 *}+r_{n j *},
$$


to which we may apply (W). Recall $r_{i k_{i}} \$ r_{i+1,0}+r_{n j}$ from above, whence $r_{i k_{i}}$ is not below the right-hand side. By property 2.1(ii), which was proved above, we have $r_{i+1,0 *} \leqslant \kappa\left(r_{n j}\right)$, so that $r_{n j} \$ r_{i+1,0 *}+r_{n j *}$; hence the second term is not below the right-hand side. Of course $r_{i+1,0} \$ r_{i+1,0 *}$, while $r_{i+1,0} \$ r_{n j *}$ by the special case done earlier. Thus if (iv) fails, then so does (W), whence we conclude that (iv) must hold. Therefore $(\dagger \dagger)$ is a $B$-type sequence, as claimed.

4. The main result. In this section we will prove that an $S$-lattice cannot contain a cycle, which combined with Theorem 1.2 shows that every $S$-lattice can be embedded in a free lattice. We will follow the plan outlined in $\$ 1$.

So suppose that $L$ is an $S$-lattice containing a cycle. Since $p A q$ implies $p>q$, it is clear that we cannot have a cycle containing only $A$ 's. By Lemma 1.3(ii), then neither can we have a cycle containing all $B$ 's, for then $L^{d}$ would contain a cycle with all $A$ 's. So let

$$
\begin{aligned}
& q_{10} B^{k_{1}} q_{1 k_{1}}=p_{10} A^{m_{1}} p_{1 m_{1}} \\
= & q_{20} B^{k_{2}} q_{2 k_{2}}=p_{20} A^{m_{2}} p_{2 m_{2}} \\
\vdots & \\
= & q_{n 0} B^{k_{n}} q_{n k_{n}}=p_{n 0} A^{m_{n}} p_{n m_{n}}=q_{10}
\end{aligned}
$$

be a minimal cycle in $L$, where $q_{10} B^{k_{1}} q_{1 k_{1}}$ means that $q_{10} B q_{11} B \ldots B q_{1 k_{1}}$, etc.

Claim 4.1. $n>1$. For suppose $n=1$. Then $k_{1}>1$, otherwise we would have $q_{10}=p_{1 m_{1}}<p_{10}=q_{11}$, whereas $q_{10} B q_{11}$ implies that these elements are incomparable. Lemma 1.3 then implies that also $m_{1}>1$, since $A$ 's and $B$ 's are interchanged in the dual cycle. If $p_{10} A_{1} p_{11}$, Lemma 3.1 applies, yielding $q_{10 *}>p_{11 *}>$ $p_{1 m_{1} *}$. If $p_{10} A_{2} p_{11}$, then, by Lemma $3.2, p_{11} A_{2} p_{12}$, so that Lemma 3.3 applies to give $q_{10 *}>p_{12 *} \geqslant p_{1 m_{1} *}$. Thus in either case $q_{10 *}>p_{1 m_{1 *}}=q_{10 *}$, a contradiction. Hence $n>1$.

Claim 4.2. The cycle contains no $A_{1}$. For suppose (wlog) that say $p_{n j} A_{1} p_{n j+1}$ for some $j$, whence by Lemma 3.2 we have $p_{n 0} A_{1} p_{n 1}$. Let us begin by considering the subsequence

$$
q_{10} B^{k_{1}} q_{1 k_{1}}=p_{10} A^{m_{1}} p_{1 m_{1}} \text {. }
$$

One of Lemmas 3.1, 3.3, 3.4 or 3.8 applies to this subsequence, with the consequence that either

(a) $q_{10 * *} \geqslant \mathrm{p}_{1 m_{1} * *}=q_{20 * *}$ or

(b) $\left\langle q_{10}, \ldots, q_{1, k_{1}-1}, q_{20}, \ldots, q_{2 k_{2}}\right\rangle$ is a $B$-type sequence. (Indeed, (a) holds unless Lemma 3.8 applies.) Now if (a) holds, we proceed to apply one of the same four lemmas to $q_{20} B^{k_{2}} q_{2 k_{2}}=p_{20} A^{m_{2}} p_{2 m_{2}}$, obtaining either

(a) $q_{20 * *} \geqslant p_{2 m_{2} * *}=q_{30 * *}$ or

(b) $\left\langle q_{20}, \ldots, q_{2, k_{2}-1}, q_{30}, \ldots, q_{3 k_{3}}\right\rangle$, is a $B$-type sequence.

Otherwise (b) holds, in which case one of the four lemmas applies to the sequence

$$
\left\langle q_{10}, \ldots, q_{1, k_{1}-1}, q_{20}, \ldots, q_{2 k_{2}}=p_{20}, \ldots, p_{2 m_{2}}\right\rangle \text {, }
$$


yielding one of the conclusions

(a) $q_{10 * *} \geqslant p_{2 m_{2 * *}}=q_{30 * *}$ or

(b)" $\left\langle q_{10}, \ldots, q_{1, k_{1}-1}, q_{20}, \ldots, q_{2, k_{2}-1}, q_{30}, \ldots, q_{3 k_{3}}\right\rangle$, is a $B$-type sequence.

Continuing in this manner, we obtain a sequence of indices $i_{1}=1<i_{2}$ $<\cdots<i_{t}<n$ (where possibly $t=1$ ) such that

(a) ${ }^{\prime \prime \prime} q_{10 * *} \geqslant q_{i_{2} 0 * *} \geqslant \cdots \geqslant q_{i_{i} 0 * *}$ and

(b) ${ }^{\prime \prime \prime}\left\langle q_{i_{t}}, \ldots, q_{j, k_{j}-1}, q_{j+1,0}, \ldots, q_{n k_{n}}\right\rangle$ is a $B$-type sequence.

At this point, since we have (b)" ${ }^{\prime \prime}$ and $q_{n k_{n}}=p_{n 0} A_{1} p_{n 1}$, Lemma 3.1 applies to yield $q_{i_{i} 0 * *}>p_{n 1 * *}>p_{n k_{n} * *}=q_{10 * *}$, contradicting (a) ${ }^{\prime \prime \prime}$. Therefore the cycle contains no $A_{1}$.

If we assume instead that $p_{n 0} A_{2} p_{n 1} A_{2} p_{n 2}$, the same arguments give (a)"' and (b) ${ }^{\prime \prime}$, whence Lemma 3.3 yields $q_{i_{i} 0 * *}>p_{n 2 * *} \geqslant p_{n k_{n} * *}=q_{10 * *}$, with the same contradiction. Hence we have also shown

Claim 4.3. The cycle contains no consecutive pair of $A_{2}$ 's.

We conclude then that $m_{j}=1$ for all $j, 1 \leqslant j \leqslant n$. Since these considerations also apply to the dual cycle, Lemma 1.3 gives us $k_{j}=1$ for all $j$. Thus we may simplify notation by relabeling the cycle

$$
q_{1} B p_{1} A_{2} q_{2} B \ldots p_{n-1} A_{2} q_{n} B p_{n} A_{2} q_{1} \text {. }
$$

Claim 4.4. Every $B$ in the cycle is a $B_{1}$. Suppose on the contrary that say $q_{n} B_{2} p_{n}$, i.e., $p_{n *}<q_{n *}$. Then as before we can obtain a sequence of indices $i_{1}=1<i_{2}$ $<\cdots<i_{t}<n$ such that

(a) ${ }^{\prime \prime \prime \prime} q_{1 * *} \geqslant q_{i_{2} * *} \geqslant \cdots \geqslant q_{i_{1 * *}}$ and

(b) ${ }^{\prime \prime \prime \prime}\left\langle q_{i}, \ldots, p_{n-1}\right\rangle$ is a $B$-type sequence.

Now either Lemma 3.4 or Lemma 3.7 applies to $\left\langle q_{i}, \ldots, p_{n-1}\right\rangle$ and $p_{n-1} A_{2} q_{n}$. If Lemma 3.4 applies, we obtain immediately $q_{i_{* * *}} \geqslant q_{n * *}$. If Lemma 3.7 applies, we must observe that $p_{n-1} \$ q_{n}+p_{n}$. For $q_{n} \leqslant p_{n-1 *} \leqslant \kappa\left(p_{n-1}\right)$, whence also $p_{n *}<$ $q_{n *} \leqslant \kappa\left(p_{n-1}\right)$. By the free star principle, $p_{n} \leqslant \kappa\left(p_{n-1}\right)$, whence $q_{n}+p_{n} \leqslant \kappa\left(p_{n-1}\right)$. Therefore by Lemma 3.7, $q_{i_{1} *}>q_{n *}$. Thus in either case we have $q_{i_{i} * *} \geqslant q_{n * *}$. But then $q_{1 * *} \geqslant q_{i_{* *}} \geqslant q_{n * *}>p_{n * *}>q_{1 * *}$, a contradiction. Therefore $q_{j} B_{1} p_{j}$ for all $j, 1 \leqslant j \leqslant n$.

At this point, we recall Lemma 7.3 of [15]: $p A_{2} q B_{1} r$ implies $p \leqslant q+r$ (cf. the proof of Lemma 3.7 above). Thus $p_{i-1} \leqslant q_{i}+p_{i}$ for all $i, 1 \leqslant i \leqslant n$, where $p_{0} \equiv p_{n}$. Also, to avoid a degenerate case in our final argument, we borrow our next claim from Lemma 8.5 of [15].

Claim 4.5. $n>2$. For suppose $q_{1} B_{1} p_{1} A_{2} q_{2} B_{1} p_{2} A_{2} q_{1}$. Then using the above remark we see that

$$
p_{1}+p_{2}=q_{1}+p_{1}=q_{2}+p_{2}
$$

whence, by $\left(\mathrm{SD}_{\vee}\right), p_{1}+p_{2}=q_{1}+q_{2}+p_{1} p_{2}$. Now $p_{1} \$ p_{2}$ since $q_{2} \leqslant p_{1}$ and $q_{2} \$ p_{2}$, so $p_{1} p_{2} \leqslant p_{1 *}$, wherefore

$$
p_{1}+p_{2} \leqslant q_{1}+p_{1 *} \leqslant \kappa\left(p_{1}\right)
$$

a contradiction.

Thus the following lemma will apply to our cycle. 
LEMMA 4.6. Let $\left\langle r_{0}, \ldots, r_{5}\right\rangle$ be a subsequence of a minimal cycle such that $r_{0} A_{2} r_{1} B_{1} r_{2} A_{2} r_{3} B_{1} r_{4} A_{2} r_{5}$. Then

(i) $r_{0} \leqslant r_{2}+r_{4}$ and

(ii) $r_{4} \$ r_{0}+r_{2}$.

Proof. As above, Lemma 7.3 of [15] gives us $r_{0} \leqslant r_{1}+r_{2}$ and $r_{2} \leqslant r_{3}+r_{4}$, whence in particular $r_{4} \$ \kappa\left(r_{2}\right)$ since $r_{3} \leqslant r_{2 *} \leqslant \kappa\left(r_{2}\right)$. By the free star principle, $r_{4 *} \$ \kappa\left(r_{2}\right)$. Now $r_{2} \neq r_{4 *}$ since $r_{3} \leqslant r_{2}$ and $r_{3} \$ r_{4 *}$. Thus if $r_{4 * *} \leqslant \kappa\left(r_{2}\right)$, we would have $r_{2} B r_{4 *}$. If $r_{4 *}=r_{5}$, this immediately contradicts the minimality of the cycle; otherwise $r_{4 *}>r_{5}$, in which case $r_{4 *} A r_{5}$ (since $r_{5}<r_{4 *}<r_{4} \leqslant \kappa\left(r_{5}\right)^{*}$ ), again shortening the cycle. Therefore $r_{4 * *} \leqslant \kappa\left(r_{2}\right)$, i.e., $r_{2} \leqslant r_{2 *}+r_{4 * *}$. It follows in particular from this last statement that $r_{4 * *} \$ r_{3 *}$, since $r_{3 *} \leqslant r_{2 *}$.

We can now prove a strong version of 4.6(ii). Note that $r_{1}<r_{0} \leqslant r_{1}+r_{2}$ implies $r_{0}+r_{2}=r_{1}+r_{2}$. Suppose $r_{4 * *} \leqslant r_{0}+r_{2}$. Then we would have $r_{1}+r_{2}=r_{1}+r_{2 *}$ $+r_{4 * *}$ whence by $\left(\mathrm{SD}_{\vee}\right)$

$$
r_{1}+r_{2}=r_{1}+r_{2 *}+r_{2} r_{4 * *}
$$

Since $r_{3} \leqslant r_{2}$ and $r_{3} \leqslant r_{4}$, we have $r_{2} \leqslant r_{4 * *}$, so that $r_{2} r_{4 * *} \leqslant r_{2 *}$. Thus $r_{1}+r_{2}=r_{1}$ $+r_{2 *} \leqslant \kappa\left(r_{2}\right)$, a contradiction. Therefore $r_{4 * *} \leqslant r_{0}+r_{2}$.

Now suppose that $4.6(i)$ fails, i.e., $r_{0} \$ r_{2}+r_{4}$. Then $\left(r_{0}+r_{2}\right)\left(r_{2}+r_{4}\right)$ is meet-reducible, and hence join-irreducible. Let us apply Lemma 2.3 with

$$
\begin{aligned}
& a=r_{2}, \quad a_{0}=\left(r_{0}+r_{2}\right)\left(r_{2}+r_{4}\right), \quad a_{1}=a_{0 *}, \\
& b=r_{3 *}+r_{4 * *}, \quad b_{0}=r_{3 *}+r_{4 *} .
\end{aligned}
$$

We must check conditions (i)' and (ii)-(vii).

(i)' Certainly $a=r_{2} \in J(L)$, and $a=r_{2} \leqslant r_{2 *}+r_{4 * *}=a_{*}+b$ by the above remarks. Also $r_{3 *} \$ r_{4 * *}$ since $r_{3} B r_{4}$, while $r_{4 * *} r_{3}$ was shown above, so $b$ is join-reducible.

(ii) Clearly $a \leqslant a_{0}$; we need to show that $a<a_{0}$, i.e., $\left(r_{0}+r_{2}\right)\left(r_{2}+r_{4}\right) \neq r_{2}$. Otherwise, we could apply (W) to the inclusion

$$
\left(r_{0}+r_{2}\right)\left(r_{2}+r_{4}\right)=r_{2} \leqslant r_{2 *}+r_{4 *} \text {. }
$$

Now $r_{0} \$ r_{2}+r_{4}$ by assumption, so the first term is not below the right-hand side. On the other hand $r_{2} \$ r_{2 *}$ and $r_{2} \$ r_{4 *}$ (since $r_{3}<r_{2}$ and $r_{3} B r_{4}$ ), which means that the second term must be below the right-hand side, i.e., $r_{4} \leqslant r_{2}+r_{4 *}$. But then, using $r_{3} \leqslant r_{2 *}<r_{2} \leqslant r_{3}+r_{4}$, we have $r_{3}+r_{4}=r_{2 *}+r_{4 *}$. Applying $\left(\mathrm{SD}_{\vee}\right)$ we obtain

$$
r_{3}+r_{4}=r_{3}+r_{4 *}+r_{2 *} r_{4} \text {. }
$$

However, $r_{4} \$ r_{2 *}$ since $r_{4 * *} \$ \kappa\left(r_{2}\right)$, so $r_{2 *} r_{4} \leqslant r_{4 *}$. Thus $r_{3}+r_{4}=r_{3}+r_{4 *} \leqslant$ $\kappa\left(r_{4}\right)$, a contradiction. Therefore $\left(r_{0}+r_{2}\right)\left(r_{2}+r_{4}\right) \neq r_{2}$, so that $a<a_{0}$ whence $a \leqslant a_{0 *}=a_{1}$, as desired.

(iii) $b \leqslant b_{0}$ is clear.

(iv) We have $r_{3}<r_{2}=a$, while $b_{0}=r_{3 *}+r_{4 *} \leqslant \kappa\left(r_{3}\right)$ since $r_{3} B r_{4}$. Hence $a \$ b_{0}$.

(v) $b \$ a_{0}$ follows from $r_{4 * *} \$ r_{0}+r_{2}$. 
(vi) If $a_{0} \leqslant a_{1}+b_{0}$, then we may apply (W) to the inclusion

$$
\left(r_{0}+r_{2}\right)\left(r_{2}+r_{4}\right)=a_{0} \leqslant a_{1}+r_{4 *} \text {, }
$$

where we have used $r_{3 *}<r_{2}=a \leqslant a_{1}$. Now $r_{0} \$ a_{1}+r_{4 *}$ since $a_{1}+r_{4 *} \leqslant r_{2}+$ $r_{4}$. On the other hand $a_{0} \$ a_{1}$, and $a_{0} \$ r_{4 *}$ since $r_{2} \$ r_{4 *}$. Therefore we must have $r_{4} \leqslant a_{1}+r_{4 *}$, whence $r_{4} \leqslant a_{0}+r_{4 *}$. But then, arguing as above, $r_{3}+r_{4}=a_{0}+$ $r_{4 *}$, whence by $\left(\mathrm{SD}_{\vee}\right)$

$$
r_{3}+r_{4}=r_{3}+r_{4 *}+r_{4} a_{0}=r_{3}+r_{4 *} \leqslant \kappa\left(r_{4}\right)
$$

since $r_{4} \$ a_{0}$ because $a_{0} \leqslant r_{0}+r_{2}$. This is a contradiction, whereupon we conclude $a_{0} \$ a_{1}+b_{0}$.

(vii) If $b_{0} \leqslant a_{1}+b$, then $r_{4 *} \leqslant a_{1}+r_{4 * *}$ (where again we have used $r_{3 *} \leqslant a_{1}$ ). Now the last argument in (vi) shows that $r_{4} \$ a_{0}+r_{4 *}$. Hence $r_{4}\left(a_{0}+r_{4 *}\right)=r_{4 *}$, so we may apply $(\mathrm{W})$ to the inclusion

$$
r_{4}\left(a_{0}+r_{4 *}\right)=r_{4 *} \leqslant a_{1}+r_{4 * *} \text {. }
$$

Of course $r_{4} \$ a_{1}+r_{4 * *}$ since $r_{4} \$ a_{0}+r_{4 *}$, while $a_{0} \$ a_{1}+r_{4 * *}$ since $a_{0} \$ a_{1}+$ $r_{4 *}$ by (vi). On the other hand, $r_{4 *} \$ a_{1}$ because $r_{4 * *} \$ r_{0}+r_{2}$, and obviously $r_{4 *} \$ r_{4 * *}$. Thus the assumption $b_{0} \leqslant a_{1}+b$ leads to a W-failure, whence $b_{0} \$ a_{1}$ $+b$.

By Lemma 2.3, this configuration cannot exist in an $S$-lattice. Therefore 4.6(i) holds, as desired.

Now let us apply Lemma 4.6 to our current situation. By repeated application of 4.6(i),

$$
p_{1} \leqslant p_{2}+p_{3} \leqslant\left(p_{3}+p_{4}\right)+p_{3}=p_{3}+p_{4} \leqslant \cdots \leqslant p_{n-1}+p_{n} .
$$

However, if we let $r_{0}=p_{n-1}, 4.6$ (ii) says that $p_{1} \$ p_{n-1}+p_{n}$. This contradiction eliminates our last possibility for the existence of a cycle in an $S$-lattice.

Thus Jónsson's conjecture is true.

THEOREM 4.7. A finite lattice is a sublattice of a free lattice iff it is semidistributive and satisfies $(W)$.

In closing, we remark that most of the problems discussed in [15] have now been solved. A notable exception to this is the problem of characterizing arbitrary sublattices of a free lattice. Distributive sublattices of a free lattice were described by Galvin and Jónsson [7], and the arguments of [15], based in large part on Kostinsky [16], show that a finitely generated lattice is embeddable in a free lattice iff it satisfies (W) and the generators are contained in $D(L) \cap D^{\prime}(L)$. Beyond this little is known except a few necessary conditions (see [15, 6]). Perhaps the countable case would be a good place to start.

\section{REFERENCES}

1. R. Antonius and I. Rival, A note on Whitman's property for free lattices, Algebra Universalis 4 (1974), 271-272.

2. B. Davey, W. Poguntke and I. Rival, A characterization of semidistributivity, Algebra Universalis 5 (1975), 72-75.

3. B. Davey and B. Sands, An application of Whitman's condition to lattices with no infinite chains, Algebra Universalis 7 (1977), 171-178. 
4. A. Day, Characterizations of lattices that are bounded-homomorphic images or sublattices of free lattices, Canad. J. Math. 31 (1979), 69-78.

5. A. Day and J. B. Nation, A note on finite sublattices of free lattices, Algebra Universalis. (to appear).

6. R. Freese and J. B. Nation, Projective lattices, Pacific J. Math. 75 (1978), 93-106.

7. F. Galvin and B. Jónsson, Distributive sublattices of a free lattice, Canad. J. Math. 13 (1961), 265-272.

8. H. Gaskill, G. Grätzer and C. R. Platt, Sharply transferable lattices, Canad. J. Math. 27 (1975), $1246-1262$.

9. H. Gaskill and C. R. Platt, Sharp transferability and finite sublattices of free lattices, Canad. J. Math. 27 (1975), 1036-1041.

10. G. Grätzer and C. R. Platt, A characterization of sharply transferable lattices, Canad. J. Math. 32 (1980), 145-154.

11. J. Jězek and V. Slavik, Primitive lattices, Czechoslovak Math. J. 29 (1979), 595-634.

12. B. Jonsson, Sublattices of a free lattice, Canad. J. Math. 13 (1961), 256-264.

13. __ Relatively free lattices, Colloq. Math. 21 (1970), 191-196.

14. B. Jónsson and J. Kiefer, Finite sublattices of a free lattice, Canad. J. Math. 14 (1962), 487-497.

15. B. Jónsson and J. B. Nation, A report on sublattices of a free lattice, Colloq. Math. Soc. János Bolyai, no. 17, Contributions to Universal Algebra (Szeged), North-Holland, Amsterdam, 1977, pp. 223-257.

16. A. Kostinsky, Projective lattices and bounded homomorphisms, Pacific J. Math. 40 (1972), 111-119.

17. H. Lakser, Finite projective lattices are sharply transferable, Algebra Universalis 5 (1975), 407-409.

18. R. McKenzie, Equational bases and non-modular lattice varieties, Trans. Amer. Math. Soc. 174 (1972), 1-43.

19. J. B. Nation, Bounded finite lattices, Colloq. Math. Soc. János Bolyai, Contributions to Universal Algebra (Esztergom), North-Holland, Amsterdam (to appear).

20. C. R. Platt, Finite transferable lattices are sharply transferable, Proc. Amer. Math. Soc. 81 (1981), $355-358$.

21. I. Rival and B. Sands, Planar sublattices of a free lattice, Canad. J. Math. 30 (1978), 1256-1283.

22. H. Rolf, The free lattice generated by a set of chains, Pacific J. Math. 8 (1958), 585-595.

23. P. M. Whitman, Free lattices, Ann. of Math. (2) 42 (1941), 325-330.

24. , Free lattices. II, Ann. of Math. (2) 43 (1942), 104-115.

Department of Mathematics, University of Hawail at Manoa, Honolulu, Hawail 96822 